\title{
Phylogenetic Relationships Between and Within 11 Taxa of Genus Vicia from Algeria Based on Evidence from Isozymes and Physical Seed Properties
}

\author{
Sakina Bechkri, \\ Imane Medoukali, \\ Douadi Khelifi,
}

Laboratoire de Génétique Biochimie et Biotechnologies Végétales. Faculté des sciences de la nature et de la vie. Université des Frères MENTOURI 25000 Constantine, Algérie

doi: 10.19044/esj.2017.v13n9p168 URL:http://dx.doi.org/10.19044/esj.2017.v13n9p168

\begin{abstract}
Isozyme patterns for aspartate amino transferase, alcohol dehydrogenase and superoxide dismutase were evaluated in 60 accessions of eleven Vicia taxa occurring in Algeria. The three enzymatic systems revealed 25 morphs of different frequency encoded by 7 loci. Similarity between species and accessions was estimated by Euclidean distances based on presence or absence of bands. The UPGMA method was utilized for the groupings and dendrogram construction. The dendrogram presented 2 major groups, each one corresponding to a taxonomic level where accessions of the same species grouped together. Taxa-specific bands were identified. In parallel, 12 physical seed properties namely: length, width, thickness, arithmetic diameter, geometric diameter, sphericity, surface area, volume, square mean diameter, equivalent diameter, seed aspect ratio and 1000 seed weight were calculated. By cluster analyses based upon them, the material has been classified into 2 major groups with no correspondence with the current taxonomy. A Principal Component Analysis was undertaken to distinguish physical properties which contribute the most to accessions distinction. Mantel test was used to assess correlation between the three enzymatic systems studied one side and between enzymes polymorphism and physical seed properties another side.
\end{abstract}

Keywords: Algeria; isozymes; seed properties; Vicia

\section{Introduction}

Vicia L. (Fabaceae) is a medium-sized genus comprising about 150 (Kupicha 1976) to 210 (Hanelt and Mettin 1989) species indicating problems 
with species circumscription and ranking. 26 species occur in Algeria, according to the flora described by Quézel and Santa (1962). The infrageneric taxonomy also poses numerous continuously disputed problems, with several competing taxonomic treatments exisiting. A through evaluation of the genetic variability present within the genus is required if Vicia material is to be utilized efficiently in plant breeding programmes. Germplasm characterization, that can be performed by several approaches such as morphology, cytogenetics, biochemistry and geographical distribution, is an essential tool for plant breeding (Gonzalez and ShiffinoWittman 1996). Allozyme polymorphism, is useful to examine genetic processes at every stage of the life cycle and to ascertain genetic diversity in plant species. Isozymes are good taxonomic characters for grouping vetch species into monophyletic sections and also provide additional diagnostic characters for distinguishing between species (Leht and Jaaska 2002). Literature data on isozymes for the genus show that some enzymatic systems allow species or species group characterization (Yamamoto and Plitmann 1980; Suso and Moreno 1986), determination of species relationships (Wolff 1980; Yamamoto 1986) as well as detection of hybrids and assessment of the degree of genetic recombination (Yamamoto 1979; Gates and Boulter 1980). The isozyme data described by Jaaska (1997) regarding monophyletic groups and their relationships are in close agreement with those based on the study of RAPDs and cpDNA RFLP by Potokina et al. (1999). Species groups revealed by isozymes by Leht and Jaaska (2002) were in a general agreement with traditionally recognized sections, contributing to the debated sectional placement of some species. Yamamoto and Plitmann (1980) studied isozymes in Vicia species without making any phylogenetic inferences. In another side, Vicia taxonomists have traditionnaly concentrated on floral and leaf morphology, paying little attention to seed characters. Iannelli (1964) discussed the need for accurate identification of seed lots due to the increasing agricultural use of vetches and he found that species could be recognized using certain seed characters. Morever, physical properties of vetches seeds are to be known for design and improve of relevant machines and facilities for handling, storing, harvesting and processing. The size and shape are important in designing of separating, sizing and granding machines (Taser et al. 2005). Various types of cleaning, grading and separation equipment are designed on the basis of the physical properties of grains or seeds. Wu et al. (1999) reported the importance of difference in size and density during separation of particles by segregating on gravity tables. Size, shape and density are important in the separation of seed from undesirable materials on oscillating chafers (Zewdu 2004; Scherer and Kutzbach 1978; Hauhouot-O ${ }^{e c}$ Hara et al. 2000). Review of literatures showed that there is not much information relating to physical properties of vetch grain from Algeria. 
Our recent studies of phylogenetic relationships in the the genus Vicia by morphology and seed storage proteins gave conflicting results especially concerning $V$. staiva s.l. (Bechkri and Khelifi 2016). The present paper describes variation of three isozymes and twelve physical seed properties in a set of eleven Algerian taxa belonging to four traditional sections of the genus Vicia in order to study the intra and interspecific diversity, to improve the resolution of phylogenetic relationships and evaluate the usefulness of the two markers as diagnostic characters to discriminate vetch species and sections.

\section{Material and methods}

\section{Plant material and taxa identification}

Sixty accessions belonging to 11 Vicia $\mathrm{L}$. taxa collected from their natural habitats in various bioclimatic conditions of Algeria are used in the current study (Figure 1). A Global Positioning Systems (GPS GARMIN eTrex ${ }^{\circledR}$ model 30 ) was used to collect latitude, longitude and altitude of sites investigated. The data on the vetch species and accessions investigated are given in Table 1. Individual plants were randomly collected in each sampling site.

Figure 1. Geographical origin of the 60 Algerian populations studied

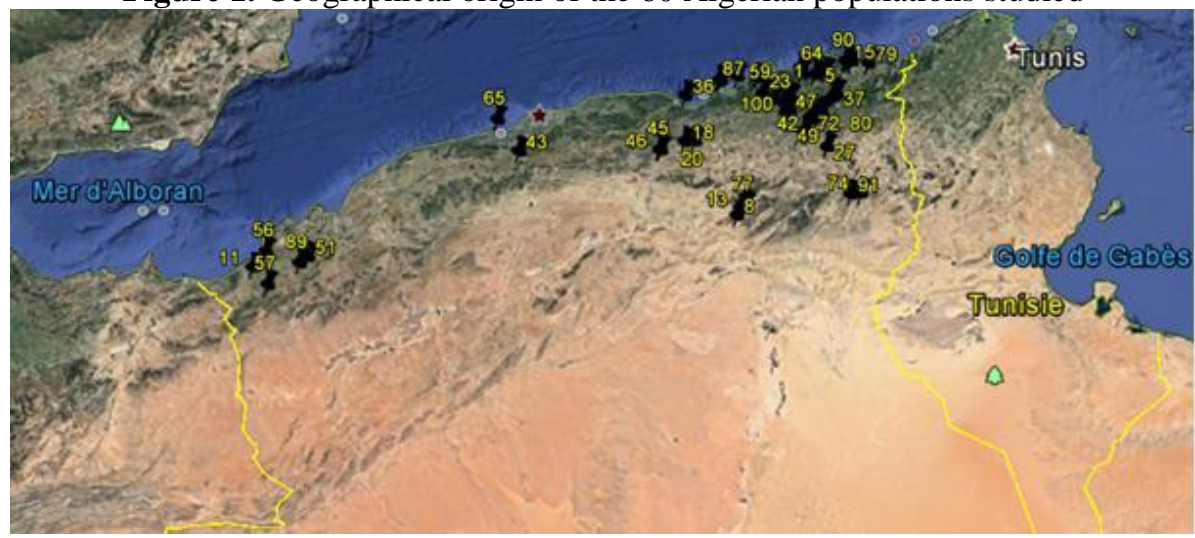

Pods were then shelled and the dry seeds poured in to separate sealed paper bags at room temperature until their utilization. Taxonomic identification of accessions was verified by the morphology of plants grown from seeds in the greenhouse of the laboratory of "Génétique Biochimie et Biotechnologies Végétales" (N 36²0.602' E006 ${ }^{\circ} 37.480^{\prime}$ Alt. 569m) of Faculty of Biology in Constantine University (eastern Algeria), using the key provided by Quézel and Santa (1962). Taxonomic nomenclature at subgeneric and sectional levels follows Kupicha (1976) and Maxted (1993). 
Table 1. Passport information and taxonomic identification of accessions investigated

\begin{tabular}{|c|c|c|c|c|c|c|}
\hline Species/Subspecies & Code & $\begin{array}{l}\text { Date of } \\
\text { collectio } \\
\mathbf{n}\end{array}$ & Province /Locality/Origin & Latitude & Longitude & $\begin{array}{l}\text { Altitude } \\
(\mathbf{m})\end{array}$ \\
\hline \multirow{8}{*}{$\begin{array}{l}\text { V. sativa subsp. } \\
\text { consobrina } \\
\text { (Pomel) Maire }\end{array}$} & 5 & 28.5 .14 & Guelma & $\mathrm{N} 36^{\circ} 26.187^{\prime}$ & E007 17.772 & 339 \\
\hline & 14 & 1st.6.14 & Annaba $\quad$ El bouni & $\mathrm{N} 36^{\circ} 49.777^{\prime}$ & E007³8.290 & 28 \\
\hline & 36 & 23.5 .14 & Affalou & $\mathrm{N} 36^{\circ} 40.381^{\prime}$ & $\mathrm{E} 005^{\circ} 08.903$ & 1 \\
\hline & 59 & 30.5 .14 & Jijel & $\mathrm{N} 36^{\circ} 35.082^{\prime}$ & $\mathrm{E}^{\mathrm{E}} 006^{\circ} 16.728$ & 141 \\
\hline & 64 & 1st.6.14 & Skikda Azzaba & $\mathrm{N} 36^{\circ} 43.532^{\prime}$ & E00704.706 & 111 \\
\hline & $65 a$ & 9.6 .14 & $\begin{array}{l}\text { Tipaza } \\
\text { mountain }\end{array}$ & $\mathrm{N} 36^{\circ} 37.667^{\prime}$ & E002²1.150 & 345 \\
\hline & 85 & 1st.6.14 & Skikda Ain Charchar & $\mathrm{N} 36^{\circ} 44.366^{\prime}$ & E007¹4.176 & 52 \\
\hline & 93 & 30.5 .14 & Jijel & $\mathrm{N} 36^{\circ} 48.699^{\prime}$ & $\begin{array}{l}\mathrm{E} 005^{\circ} 41.679 \\
\end{array}$ & 25 \\
\hline \multirow{13}{*}{$\begin{array}{l}\text { V. sativa subsp. } \\
\text { obovata Gaudin }\end{array}$} & 6 & 22.5 .14 & Constantine & $\mathrm{N} 36^{\circ} 20.628^{\prime}$ & E006 37.485 & 563 \\
\hline & 7 & 30.5 .14 & Messaoud Boudjriou & $\mathrm{N} 36^{\circ} 29.743^{\prime}$ & E006 25.527 & 325 \\
\hline & 10 & 27.5 .14 & Didouche Mourad & $\mathrm{N} 36^{\circ} 28.409^{\prime}$ & $\begin{array}{l}\text { E006 } 38.239 \\
\end{array}$ & 468 \\
\hline & 17 & 22.5 .14 & Constantine & $\mathrm{N} 36^{\circ} 20.628^{\prime}$ & $\begin{array}{l}\text { E006 } 37.485 \\
\end{array}$ & 563 \\
\hline & 20 & 3.6.14 & Ain arnat & $\mathrm{N} 36^{\circ} 07.394^{\prime}$ & E005¹2.172 & 866 \\
\hline & 22 & 2.6 .14 & Oum El Bouaghi $\quad$ Sigus & $\mathrm{N} 36^{\circ} 04.485^{\prime}$ & E006 48.867 & 822 \\
\hline & 51 & 6.6 .14 & Sidi Bel Abbes & $\mathrm{N} 35^{\circ} 10.824^{\prime}$ & $\begin{array}{l}\text { W000³6.02 } \\
6^{\prime}\end{array}$ & 490 \\
\hline & 32 & 22.5 .14 & Constantine Chaab Ersas & $\mathrm{N} 36^{\circ} 20.634^{\prime}$ & E006³7.486 & 562 \\
\hline & 57 & 6.6 .14 & Tlemcen $\quad$ Ain fezza & $\mathrm{N} 34^{\circ} 52.732^{\prime}$ & $\begin{array}{l}\text { W001¹3.72 } \\
6^{\prime}\end{array}$ & 867 \\
\hline & 72 & 28.5 .14 & Constantine Ain abid & $\mathrm{N} 36^{\circ} 13.543^{\prime}$ & E006 55.782 & 847 \\
\hline & 80 & 28.5 .14 & Constantine $\quad$ Ain abid & $\mathrm{N} 36^{\circ} 13.543^{\prime}$ & E00655.782 & 847 \\
\hline & 70 & 28.5.15 & Guelma & $\mathrm{N} 36^{\circ} 14.816^{\prime}$ & E00703.045 & 757 \\
\hline & 83 & 26.5.14 & Batna Ain Touta & $\mathrm{N} 35^{\circ} 17.632^{\prime}$ & E00549.035 & 683 \\
\hline \multirow[t]{3}{*}{$\begin{array}{l}\text { V. sativa subsp. } \\
\text { angustifolia (L.) } \\
\text { Gaudin }\end{array}$} & 19 & 18.5.14 & Chaab Ersas & $\mathrm{N} 36^{\circ} 20.634^{\prime}$ & E006³7.486 & 562 \\
\hline & 8 & 26.5.14 & El Kantra & $\mathrm{N} 35^{\circ} 11.517^{\prime}$ & E00540.673 & 467 \\
\hline & 11 & 6.6 .14 & Tlemcen & $\mathrm{N} 35^{\circ} 05.699^{\prime}$ & $\begin{array}{l}\text { W001²6.61 } \\
2^{\prime}\end{array}$ & 90 \\
\hline \multirow[b]{3}{*}{$\begin{array}{l}\text { V. sativa subsp. } \\
\text { cordata (Will) } \\
\text { Batt. }\end{array}$} & 13 & 26.5 .14 & $\begin{array}{lll}\text { Biskra } & \text { El Kantra } & \text { Ain } \\
\text { Skhoun } & & \end{array}$ & $\mathrm{N} 35^{\circ} 16.087^{\prime}$ & E00544.174 & 584 \\
\hline & 15 & 1st.6.14 & Annaba & $\mathrm{N} 36^{\circ} 49.980^{\prime}$ & E007³4.092 & 24 \\
\hline & 33 & 29.5.14 & El hadaik & $\mathrm{N} 36^{\circ} 49.894^{\prime}$ & E00653.079 & 26 \\
\hline
\end{tabular}




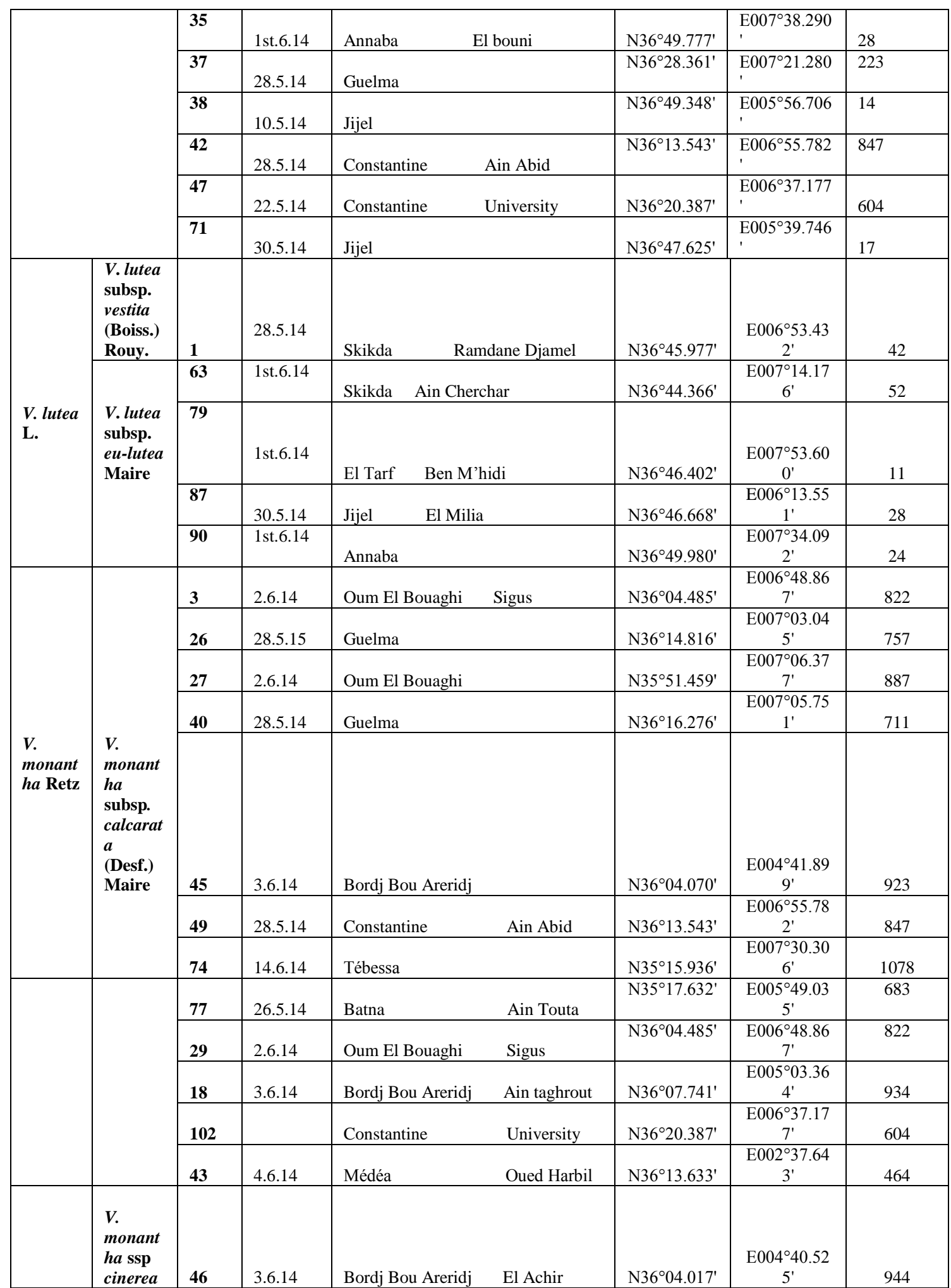




\begin{tabular}{|c|c|c|c|c|c|c|c|}
\hline & $\begin{array}{l}\text { (M.B.) } \\
\text { Maire }\end{array}$ & & & & & & \\
\hline & & 91 & 14.6 .14 & Khenchla & $\mathrm{N} 35^{\circ} 15.704^{\prime}$ & $\begin{array}{c}\mathrm{E} 007^{\circ} 20.95 \\
7^{\prime} \\
\end{array}$ & 1222 \\
\hline \multirow{5}{*}{$\begin{array}{l}\text { V. } \\
\text { narbon } \\
\text { ensis L. }\end{array}$} & \multirow{5}{*}{ - } & 23 & 30.5 .14 & $\begin{array}{l}\text { Constantine Hamma Bouziane } \\
\text { (Chaabet El Medhbouh) }\end{array}$ & $\mathrm{N} 36^{\circ} 26.391^{\prime}$ & $\begin{array}{c}\text { E006 } 33.28 \\
2^{\prime} \\
\end{array}$ & 425 \\
\hline & & 34 & 27.4 .14 & Constantine Didouche Mourad & $\mathrm{N} 36^{\circ} 29.216^{\prime}$ & $\begin{array}{c}\mathrm{E} 006^{\circ} 38.73 \\
1^{\prime} \\
\end{array}$ & 434 \\
\hline & & 41 & 28.5 .14 & Guelma & $\mathrm{N} 36^{\circ} 16.276^{\prime}$ & $\begin{array}{c}\mathrm{E} 007^{\circ} 05.75 \\
1^{\prime} \\
\end{array}$ & 711 \\
\hline & & 55 & 27.5 .14 & Constantine Didouche Mourad & N36 $30.023^{\prime}$ & $\begin{array}{c}\text { E006 } 40.05 \\
1^{\prime}\end{array}$ & 443 \\
\hline & & 81 & 22.5 .14 & Constantine University & $\mathrm{N} 36^{\circ} 20.387^{\prime}$ & $\begin{array}{c}\text { E006 } 37.17 \\
7^{\prime}\end{array}$ & 604 \\
\hline \multirow[b]{2}{*}{$\begin{array}{l}\text { V. } \\
\text { tenuifol } \\
\text { ia } \\
\text { Roth. }\end{array}$} & \multirow[b]{2}{*}{ - } & 56 & 6.6.14 & Ain Temouchent & $\mathrm{N} 35^{\circ} 16.476^{\prime}$ & $\begin{array}{c}\text { W001 }{ }^{\circ} 13.80 \\
0^{\prime}\end{array}$ & 276 \\
\hline & & 89 & 6.6 .14 & Sidi Bel Abbes Sidi Khaled & $\mathrm{N} 35^{\circ} 06.59^{\prime}$ & $\begin{array}{c}\mathrm{W} 000^{\circ} 44.23 \\
8^{\prime}\end{array}$ & 543 \\
\hline $\begin{array}{l}\text { V. } \\
\text { leucant } \\
\text { ha Biv. }\end{array}$ & - & 100 & 10.6 .14 & Constantine & $\mathrm{N} 36^{\circ} 19.002^{\prime}$ & $\begin{array}{c}\text { E006³4.62 } \\
6^{\prime}\end{array}$ & 586 \\
\hline
\end{tabular}

\section{Seed germination}

Seed were germinated on Wattman paper in Petri dishes at $28^{\circ} \mathrm{C}$, after mechanical scarification. Seedlings were transplanted to pots with garden soil, sand and compost in the greenhouse, where the plants were grown.

\section{Enzymes polymorphism}

Preliminary tests were made with eight enzymatic systems: aspartate amino transferase (AAT), alcohol dehydrogenase (ADH), esterase (EST), malate dehydrogenase (MDH), malic enzyme (ME), amylase (AMY), peroxidase (PRX) and superoxide dismutase (SOD). AAT (E.C 2.6.1.1), SOD (E.C 1.15.1.1) and ADH (E.C 1.1.1.1) were selected for further analysis due to good resolution, polymorphism and repeatability of results.

\section{Enzymes extraction}

Extractions were made from $10 \mathrm{mg}$ of newly-opened, healthy, freshly-harvested leaflets, crushed, on ice, in $600 \mu \mathrm{L}$ of extracting solution (Jaaska 2005). The front line was monitored with bromophenol blue.

\section{Polyacrylamide Gel Electrophoresis (PAGE) and staining}

Electrophoresis was undertaken in vertical polyacrylamide gel slabs in the anodal direction in an ice-refrigerated plexiglass apparatus by applying a pulsed current of $40 \mathrm{~mA}$ and $1200 \mathrm{~V}$ per gel for about $2 \pm 3 \mathrm{~h}$ until the marker dye, bromophenol blue, reached the gel end. After electrophoresis, 
the gels were stained for isoenzymes by applying standard histochemical methods (Wendel and Weeden 1989).

\section{Physical seeds properties}

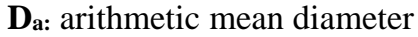
Dsq: square mean diameter

L: length of seed, mm

S: surface area, $\mathrm{mm}^{2}$

V: unit volume, $\mathrm{mm}^{3}$

$\phi:$ sphericity of seed

$D_{\text {ge: }}$ geometric mean diameter

$\mathbf{D}_{\mathbf{e}}$ : equivalent diameter $\mathbf{m}_{1000}$ : thousand seed mass, $\mathrm{g}$

T: thickness of seed, $\mathrm{mm}$

W: width of seed, mm

$\mathbf{R}_{\text {as: }}$ seed aspect ratio

Seeds were cleaned manually. Immature and broken seeds were removed. The one thousand seed mass was determined by means of an electronic balance (Denver instrument company AA-250) reading to 0.0001 g. To determine the average size of the seed, 10 seeds were randomly picked and their three linear dimensions namely, length $\mathrm{L}$, width $\mathrm{W}$ and thickness $\mathrm{T}$ were measured using a digital caliper $0-150 \mathrm{~mm}\left(6^{\prime \prime}\right)$ reading to $0.01 \mathrm{~mm}$. The average diameter of grain was calculated using the arithmetic mean and geometric mean of the three axial dimensions by the following relationships (Mohsenin 1970):

$D a=(L+W+T) / 3$

$D g=(\mathrm{LWT})^{1 / 3}$

The sphericity of seeds was calculated by using the following relationship (Mohsenin, 1970):

$\phi=(\mathrm{LWT})^{1 / 3}$

$\mathrm{L}$

The surface area of seeds was found by analogy with a sphere of same geometric mean diameter using the following expression cited by Olajide and Ade-Omowaye (1999):

$S=\pi \cdot D^{2}$

The seed volume was calculated according to Subukola and Onwuka (2011) as follow:

$V=(\pi \cdot L W T) / 6$

The square mean and equivalent diameters were determined using the formulae of Asoegwu et al. (2006):

Dsq $=((\mathrm{LW}+\mathrm{WT}+\mathrm{LT}) / 3)^{1 / 2}$

$\mathrm{D}_{\mathrm{e}}=(\mathrm{Da}+\mathrm{Dg}+\mathrm{Dsq}) / 3$

Finally, the seed aspect ratio was determined by the equation used by Seifi and Alimardani (2010):

Ras $=W / L$ 


\section{Data analyses}

The gels were scanned using imagescannIII. Bands were designated by capital letters followed by numbers, indicating the electrophoretic mobility in a scale 0-100. The isoenzyme data matrix was made using allozymes as binary absence/presence characters. Euclidean distances and UPGMA (Unweighted Pair-Group Method using Arithmetic Averages) clustering method were used to estimate isoenzymatic variation among the taxa and accessions. The relationships among the studied taxa were demonstrated as a dendrogram provided by statistical programs (STATISTICA version 6.1 program). The same method was used to construct the dendrogram of physical seed properties. To clarify relationships between the twelve physical seed properties studied and to identify which properties contribute the most in accessions separation, a Principal Component Analysis (PCA) was carried out. To display a possible correlation between the three enzymatic systems one side and between the enzymatic data and seed properties results another side, a Mantel test (Mantel 1967) based on Pearson's correlation was used (XLSTAT Pearson edition, version 2014.5.03).

\section{Results}

Data on the isoenzyme variation among the Vicia species are compiled in Table 2 where electromorphs are given in the order of their decreasing occurrence among the accessions analysed. In total, the three enzymatic systems revealed 25 morphs of different frequency encoded by 7 loci and showed variability within and between species.

Table 2. Polymorphism of Aspartate AminoTransferase (AAT), SuperOxide Dismutase

(SOD) and Alcohol DeHydrogenase (ADH) resolved from the leaflets of the Vicia taxa studied

\begin{tabular}{|c|c|c|c|c|c|c|c|c|}
\hline Taxon & $\mathbf{N}$ & AAT-B & $\begin{array}{c}\text { AAT- } \\
\text { A }\end{array}$ & SOD-C & $\begin{array}{c}\text { SOD- } \\
\text { B }\end{array}$ & SOD-A & $\underset{\text { B }}{\text { ADH }}$ & ADH A \\
\hline $\begin{array}{l}\text { V. sativa susbsp. } \\
\text { obovata }\end{array}$ & 13 & 33.8 & 43.6 & $\begin{array}{c}34.5-37.5-39.5- \\
41\end{array}$ & 46.5 & - & $\begin{array}{l}25.8- \\
29.6-\end{array}$ & $31.6-39.2$ \\
\hline $\begin{array}{l}\text { V. sativa susbsp. } \\
\text { cordata }\end{array}$ & 11 & 33.8 & $\begin{array}{r}43.6- \\
45.2\end{array}$ & $34.5-37.5$ & 46.5 & - & $\begin{array}{l}25.8- \\
29.6-\end{array}$ & 39.2 \\
\hline $\begin{array}{l}\text { V. sativa susbsp. } \\
\text { consobrina }\end{array}$ & 8 & 33.8 & 43.6 & $34.5-37.5$ & 46.5 & - & $\begin{array}{l}25.8- \\
29.6-\end{array}$ & 39.2 \\
\hline $\begin{array}{l}\text { V. sativa susbsp. } \\
\text { angustifolia }\end{array}$ & 1 & 33.8 & 43.6 & $34.5-37.5$ & 46.5 & - & $\begin{array}{l}25.8- \\
29.6-\end{array}$ & 39.2 \\
\hline $\begin{array}{l}\text { V. lutea subsp. eu- } \\
\text { lutea }\end{array}$ & 4 & 33.8 & 43.6 & 41 & 44.6 & 52.3 & $25.8-$ & $37.2-44.2$ \\
\hline V. lutea subsp. vestita & 1 & 33.8 & 45.2 & 41 & 44.6 & 52.3 & $25.8-$ & $37.2-44.2$ \\
\hline $\begin{array}{l}\text { V. monantha subsp. } \\
\text { calcarata }\end{array}$ & 12 & $27.6-31-$ & 43.6 & $34.5-41$ & 44.6 & - & $25.8-$ & 37.2 \\
\hline $\begin{array}{l}\text { V. monantha subsp. } \\
\text { cinerea }\end{array}$ & 2 & $\begin{array}{c}27.6-33.8- \\
38.2 \mathrm{r}\end{array}$ & 43.6 & $41-41.6 r$ & 44.6 & $52.3-58 \mathrm{r}$ & 25.8 & $\begin{array}{c}33.5 r-37.2- \\
44.2\end{array}$ \\
\hline V. narbonensis & 5 & 33.8 & 43.6 & 37.5 & 44.6 & 50.3 & $25.8-$ & $37.2-44.2$ \\
\hline
\end{tabular}




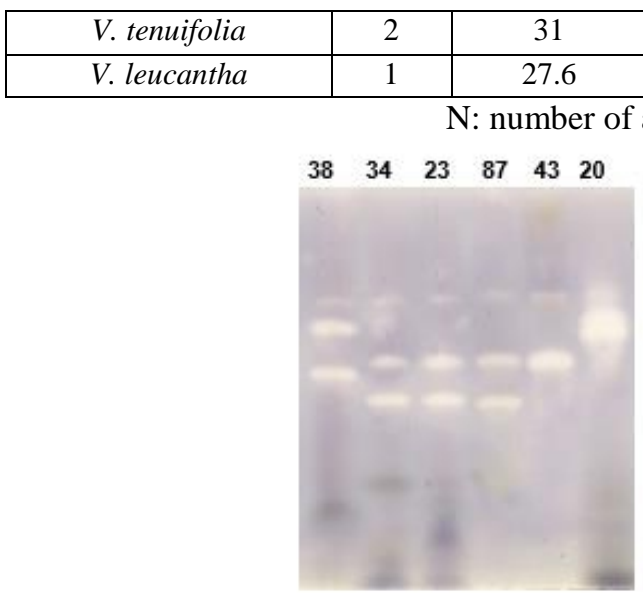

a

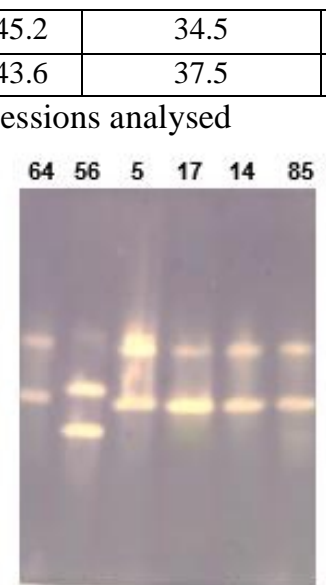

b

\begin{tabular}{|l|l|l|l|}
44.6 & 52.3 & $25.8-$ & $37.2-44.2$ \\
\hline 44.6 & $49.2 \mathrm{r}$ & $25.8-$ & $37.2-43 \mathrm{r}$ \\
\hline
\end{tabular}

r: rare morph

Figure 2. Electrophoretic banding pattern generated by PAGE of the enzymatic systems studied of some Vicia accessions

(a) $\mathrm{ADH}$

(b) SOD

(c) AAT

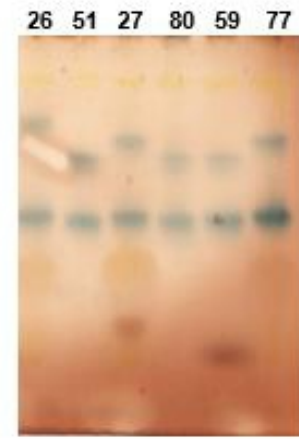

c

\section{Superoxide dismutase (SOD EC. 1.15.1.1)}

SOD zymogramms of some samples are showin in Figure $2 \mathrm{~b}$. In the present results, 11 morphs were obtained with 4 morphs for SOD-A, 2 for SOD-B and 5 for SOD-C. Each pattern contains from 2 to 3 bands. The band S34.5 is the most common as it appears in 42 profiles, followed by the band S37.5 observed in 35 profiles and bands S44.6, S46.5 observed each in 27 patterns. Three rare morphs are observed: S41.6 and S58 are specific of sample 46, when the band S49.2 is specific of sample 100. The band S50.3 is specific of 5 samples $(23,34,41,55,81)$. The band S39.5 is found in 6 patterns $(7,10,20,22,51,57)$. Accessions $5,14,36,59,64,65,85,6,17$, $32,72,80,708319811,15,33,35,37,38,42,47,71$ are characterized by a unique profile which contains the three following bands: S34.5, S37.5, S46.5. The following samples are also characterized by a unique pattern: 3693, 7-10, 20-22-51-57, 1-63-79-87-90-102, 3-26-27-40-45-49-74-77-29-18$43,23-34-41-55-81,56-89$. Samples 46 and 100 have a unique profile each.

\section{Alcohol dehydrogenase (ADH E.C 1.1.1.1)}

ADH zymogramms are shown in Figure 2a. 8 morphs were obtained for this enzymatic system. Each pattern contains from 2 to 3 bands. The band AD25.8 is the most common as it appears in 47 profiles, followed by the band AD29.6 observed in 31 profiles, band AD37.2 observed in 27 patterns and the band 39.2 observed in 25 profiles. Two rare morphs are observed: AD33.5 is specific of sample 46, when the band AD43 is specific of sample 100 . The band S50.3 is specific of 5 samples $(23,34,41,55,81)$. The band AD31.6 is found in 4 patterns $(20,22,51,57)$. Accessions $6,8,11,13,14$, $17,36,64,85,93$ are characterized by a unique profile which contains the 
two following bands: AD29.6 and AD39.2. The following samples are also characterized by a unique pattern: 1-63-79-87-90, 7-10, 19-32-33-35-37-3842-47-59-65-70-71-72-80-83, 20-22-51-57, 3-18-26-27-29-40-43-45-49-7477-91-102, 23-34-41-55-56-81-89. The sample 46 with bands AD33.5, AD37.2, AD44.2 and the sample 100 with bands AD25.8, AD37.2, AD43 have a unique profile each.

\section{Aspartate Amino Transferase (AAT E.C 2.6.1.1).}

The figure 2c shows AAT zymogramms of some samples. In the present results, 6 morphs were obtained with 2 morphs for AAT-A and 4 morphs for AAT-B. Each pattern contains 2 bands except for samples 11, 14, 36, 64, 85 and 93 where no bands have been observed for the AAT system. The band A43.6 is the most common as it appears in 47 profiles, followed by the band A33.8 observed in 38 profiles. One rare morph (A38.2) is specific of sample 46. The band A27.6 is observed in 4 samples $(3,26,91,100)$. The band A45.2 is specific of 6 samples $(1,8,13,33,56,89)$. Accessions 5-5965-6-7-10-17-19-20-22-32-51-57-72-80-70-83-35-37-38-42-47-71-79-81-8790-23-34-55-63-15-41 are characterized by a unique profile which contains the two following bands: A33.8 and A43.6. The following samples are also characterized by a unique pattern: 1-8-13-33, 3-26-91-100, 18-27-29-40-4345-49-74-77-102, 56-89. Sample 46 have a unique profile.

\section{Cluster analysis based on isozyms data}

Dendrogram generated from the three enzymatic systems is shown in figure 3. At the distance of 3.08, the dendrogram shows two major clusters. The first one is divided into two subclusters. Ia $(\mathrm{d}=2.00)$ comprises samples 57, 51, 22, 20, 10 and 7. Ib comprises two groups. The first one (Ib1) contains samples 93, 36, 85, 64, 14 and 11. The second one (Ib2) is composed of samples 33, 13 and 8 linked to accessions 83, 80, 72, 71, 70, $65,59,47,42,38,37,35,32,19,17$ and 6 also connected to samples 5 and 15. The cluster II $(\mathrm{d}=3.05)$ is divided into two subclusters. The accession 46 beign it self the first subcluster (IIa). IIb can further be divided into two groups where sample 100 forms the first group (IIb1). IIb2 comprises a first group in which samples 56 and 89 are linked to accession 102 one side and samples 77, 74, 49, 45, 43 40, 29, 27, 18 and aceesions 26 and 3 another side. A second group is composed of samples 81, 55, 41, 34 and 23 linked to the sample 91 and clustered to accessions 90, 87, 79, 63 one side and sample 1 another side. The higher distance $(\mathrm{d}=3.74)$ is observed between the sample 46 and the following accessions: 19, 20, 22, 32, 33, 35, 37, 38, 47, 51, 57 , $59,65,70,71,72,80$ and 83 . 


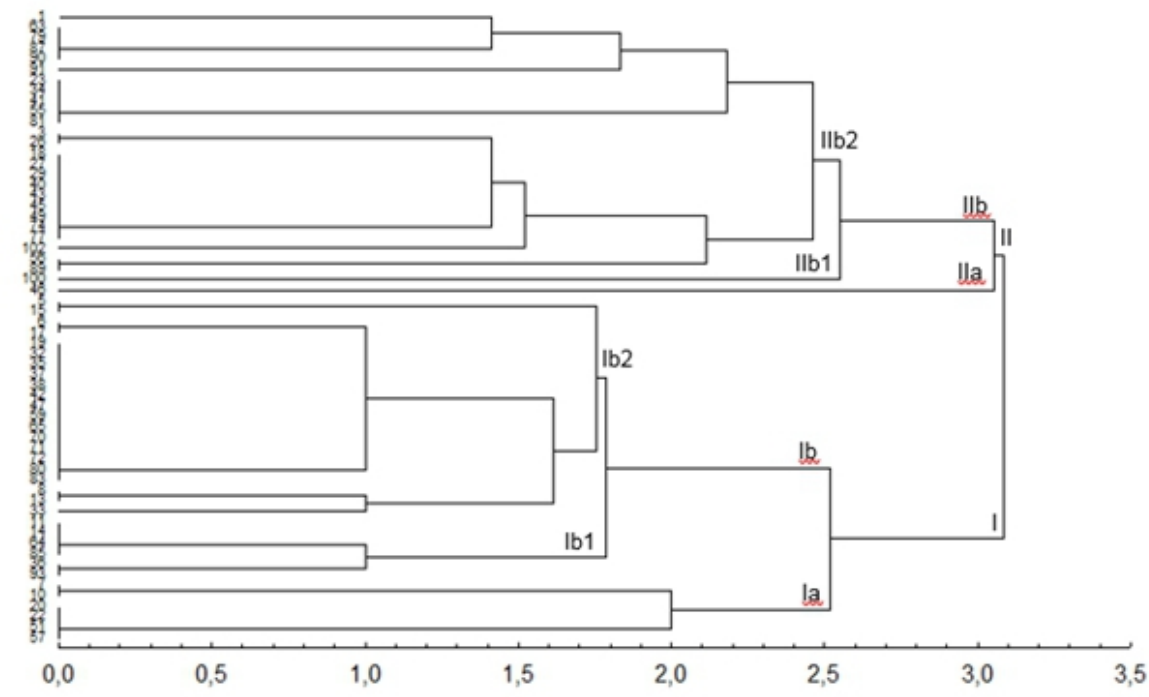

Figure 3. Dendrogram generated using UPGMA cluster analysis and Euclidean distances based on three enzymatic systems diversity of 60 Vicia accessions

\section{Correlation between enzymes patterns of the three enzymatic systems studied}

Correlation between enzymatic systems taken two by two was carried out with Mantel test based on Pearson's correlation. The p-value was calculated from the distribution of $\mathrm{r}(\mathrm{AB})$ using 10000 permutations. Correlation between SOD (matrix B) and ADH (matrix C) gives a value of $\mathrm{r}(\mathrm{AB})=0.7075$, between SOD and AAT (matrix A), $\mathrm{r}=01867$ and between $\mathrm{ADH}$ and AAT, $\mathrm{r}=0.2354$. This test shows a strong correlation between each couple of enzymatic systems, since the calculated p-values $(<0,0001)$ are below the significance level of alpha $(0.05=5 \%)$. The correlation between the three systems gives a vlaue of $\mathrm{r}(\mathrm{AB} . \mathrm{C})=0.6950$ showing also a strong correlation between the three systems studied since the calculated p-value $(<$ $0,0001)$ is below the significance level of alpha $(0.05=5 \%)$.

\section{Physical seed properties}

The physical properties of vetch, along with means and standard deviations are presented in Table 3.

Table 3. Means and standard deviations of physical seed properties of accessions studied

\begin{tabular}{|c|c|c|c|c|c|c|c|c|c|c|c|c|}
\hline $\begin{array}{c}\text { Samp } \\
\text { le }\end{array}$ & $\begin{array}{c}\mathrm{L} \\
(\mathrm{mm} \\
)\end{array}$ & $\begin{array}{c}\mathrm{W} \\
(\mathrm{mm}\end{array}$ & $\begin{array}{c}\mathrm{T} \\
(\mathrm{mm}\end{array}$ & $\mathrm{Da}$ & $\mathrm{Dg}$ & $\phi(\%)$ & $\begin{array}{c}\mathrm{S} \\
\left(\mathrm{mm}^{2}\right)\end{array}$ & $\begin{array}{c}\mathrm{V} \\
\left(\mathrm{mm}^{3}\right)\end{array}$ & Dsq & De & $\begin{array}{c}\text { Ras } \\
\mathrm{m}_{1000} \\
(\mathrm{~g})\end{array}$ \\
\hline 1 & 5,41 & 5,59 & 3,89 & 4,96 & 4,88 & 90,25 & 75,63 & 62,83 & 4,93 & 4,92 & 1,03 & \\
& \pm & \pm & \pm & \pm & \pm & \pm & \pm & \pm & \pm & \pm & \pm & \pm \\
& 0,24 & 0,91 & 0,66 & 0,50 & 0,52 & 8,723 & 16,68 & 21,52 & 0,51 & 0,51 & 0,15 & 90,33 \\
& 94 & 73 & 94 & 98 & 04 & 6 & 30 & 46 & 36 & 42 & 58 & \pm \\
& & & & & & & & & & & & 7.03 \\
\hline
\end{tabular}




\begin{tabular}{|c|c|c|c|c|c|c|c|c|c|c|c|c|}
\hline 3 & $\begin{array}{c}5,49 \\
\pm \\
0,25 \\
97\end{array}$ & $\begin{array}{c}4,53 \\
\pm \\
0,55 \\
44\end{array}$ & $\begin{array}{c}4,44 \\
\pm \\
0,20 \\
10\end{array}$ & $\begin{array}{c}4,82 \\
\pm \\
0,33 \\
84\end{array}$ & $\begin{array}{c}4,79 \\
\pm \\
0,21 \\
05\end{array}$ & $\begin{array}{c}87,33 \\
\pm \\
3,931 \\
9\end{array}$ & $\begin{array}{c}72,11 \\
\pm \\
6,388 \\
1\end{array}$ & $\begin{array}{c}57,73 \\
\pm \\
7,727 \\
0\end{array}$ & $\begin{array}{c}4,80 \\
\pm \\
0,20 \\
87\end{array}$ & $\begin{array}{c}4,80 \\
\pm \\
0,20 \\
86\end{array}$ & $\begin{array}{c}0,83 \\
\pm \\
0,11 \\
17\end{array}$ & $\begin{array}{c}78,75 \\
\pm \\
2,443 \\
2\end{array}$ \\
\hline 5 & $\begin{array}{c}4,37 \\
\pm \\
0,55 \\
81\end{array}$ & $\begin{array}{c}3,84 \\
\pm \\
0,47 \\
55\end{array}$ & $\begin{array}{c}4,08 \\
\pm \\
0,48 \\
16\end{array}$ & $\begin{array}{c}4,07 \\
\pm \\
0,37 \\
72\end{array}$ & $\begin{array}{c}3,99 \\
\pm \\
0,38 \\
48\end{array}$ & $\begin{array}{c}92,02 \\
\pm \\
7,990 \\
3\end{array}$ & $\begin{array}{c}50,55 \\
\pm \\
9,595 \\
0\end{array}$ & $\begin{array}{c}34,20 \\
\pm \\
9,624 \\
8\end{array}$ & $\begin{array}{c}4,03 \\
\pm \\
0,37 \\
97\end{array}$ & $\begin{array}{c}4,03 \\
\pm \\
0,38 \\
03\end{array}$ & $\begin{array}{c}1,08 \\
\pm \\
0,09 \\
01\end{array}$ & $\begin{array}{c}25,46 \\
\pm \\
4,084 \\
2\end{array}$ \\
\hline 6 & $\begin{array}{c}6,23 \\
\pm \\
0,32 \\
73\end{array}$ & $\begin{array}{c}5,49 \\
\pm \\
0,49 \\
63\end{array}$ & $\begin{array}{c}4,30 \\
\pm \\
0,45 \\
80\end{array}$ & $\begin{array}{c}5,34 \\
\pm \\
0,17 \\
03\end{array}$ & $\begin{array}{c}5,26 \\
\pm \\
0,16 \\
94\end{array}$ & $\begin{array}{c}84,63 \\
\pm \\
4,458 \\
6\end{array}$ & $\begin{array}{c}87,11 \\
\pm \\
5,607 \\
3\end{array}$ & $\begin{array}{c}76,55 \\
\pm \\
7,393 \\
4\end{array}$ & $\begin{array}{c}5,30 \\
\pm \\
0,16 \\
72\end{array}$ & $\begin{array}{c}5,30 \\
\pm \\
0,16 \\
71\end{array}$ & $\begin{array}{c}0,88 \\
\pm \\
0,07 \\
67\end{array}$ & $\begin{array}{c}104,8 \\
2 \pm \\
4,247 \\
7\end{array}$ \\
\hline 7 & $\begin{array}{c}4,08 \\
\pm \\
0,23 \\
45\end{array}$ & $\begin{array}{c}4,17 \\
\pm \\
0,27 \\
21\end{array}$ & $\begin{array}{c}3,35 \\
\pm \\
0,23 \\
01\end{array}$ & $\begin{array}{c}3,87 \\
\pm \\
0,18 \\
53\end{array}$ & $\begin{array}{c}3,84 \\
\pm \\
0,18 \\
53\end{array}$ & $\begin{array}{c}94,36 \\
\pm \\
4,484 \\
7\end{array}$ & $\begin{array}{c}46,52 \\
\pm \\
4,403 \\
0\end{array}$ & $\begin{array}{c}29,93 \\
\pm \\
4,169 \\
0\end{array}$ & $\begin{array}{c}3,86 \\
\pm \\
0,18 \\
52\end{array}$ & $\begin{array}{c}3,86 \\
\pm \\
0,18 \\
52\end{array}$ & $\begin{array}{c}1,29 \\
\pm \\
0,07 \\
04\end{array}$ & $\begin{array}{c}41,06 \\
\pm \\
1,260 \\
2\end{array}$ \\
\hline 8 & $\begin{array}{c}3,55 \\
\pm \\
0,25 \\
07\end{array}$ & $\begin{array}{c}3,44 \\
\pm \\
0,22 \\
92\end{array}$ & $\begin{array}{c}3,07 \\
\pm \\
0,26 \\
52\end{array}$ & $\begin{array}{c}3,35 \\
\pm \\
0,21 \\
01\end{array}$ & $\begin{array}{c}3,34 \\
\pm \\
0,21 \\
33\end{array}$ & $\begin{array}{c}94,38 \\
\pm \\
3,289 \\
8\end{array}$ & $\begin{array}{c}35,23 \\
\pm \\
4,474 \\
6\end{array}$ & $\begin{array}{c}19,77 \\
\pm \\
3,754 \\
2\end{array}$ & $\begin{array}{c}3,35 \\
\pm \\
0,21 \\
15\end{array}$ & $\begin{array}{c}3,35 \\
\pm \\
0,21 \\
16\end{array}$ & $\begin{array}{c}0,97 \\
\pm \\
0,06 \\
25\end{array}$ & $\begin{array}{c}24,96 \\
\pm \\
1,563 \\
1\end{array}$ \\
\hline 10 & $\begin{array}{c}4,70 \\
\pm \\
0,25 \\
05\end{array}$ & $\begin{array}{c}5,31 \\
\pm \\
0,48 \\
96\end{array}$ & $\begin{array}{c}3,94 \\
\pm \\
0,26 \\
37\end{array}$ & $\begin{array}{c}4,65 \\
\pm \\
0,23 \\
75\end{array}$ & $\begin{array}{c}4,61 \\
\pm \\
0,23 \\
51\end{array}$ & $\begin{array}{c}98,13 \\
\pm \\
2,768 \\
3\end{array}$ & $\begin{array}{c}66,86 \\
\pm \\
6,735 \\
0\end{array}$ & $\begin{array}{c}11,14 \\
\pm \\
1,122 \\
5\end{array}$ & $\begin{array}{c}4,63 \\
\pm \\
0,23 \\
60\end{array}$ & $\begin{array}{c}4,63 \\
\pm \\
0,23 \\
60\end{array}$ & $\begin{array}{c}1,13 \\
\pm \\
0,10 \\
75\end{array}$ & $\begin{array}{c}67,27 \\
\pm \\
4,213 \\
4\end{array}$ \\
\hline 11 & $\begin{array}{c}3,71 \\
\pm \\
0,42 \\
85\end{array}$ & $\begin{array}{c}3,70 \\
\pm \\
0,49 \\
68\end{array}$ & $\begin{array}{c}3,46 \\
\pm \\
0,50 \\
02\end{array}$ & $\begin{array}{c}3,62 \\
\pm \\
0,36 \\
28\end{array}$ & $\begin{array}{c}3,61 \\
\pm \\
0,36 \\
75\end{array}$ & $\begin{array}{c}97,52 \\
\pm \\
5,532 \\
7\end{array}$ & $\begin{array}{c}41,22 \\
\pm \\
8,103 \\
1\end{array}$ & $\begin{array}{c}6,87 \pm \\
1,350 \\
5\end{array}$ & $\begin{array}{c}3,61 \\
\pm \\
0,36 \\
49\end{array}$ & $\begin{array}{c}3,61 \\
\pm \\
0,36 \\
50\end{array}$ & $\begin{array}{c}1,00 \\
\pm \\
0,08 \\
76\end{array}$ & $\begin{array}{c}33,84 \\
\pm \\
2,322 \\
6\end{array}$ \\
\hline 13 & $\begin{array}{c}3,60 \\
\pm \\
0,13 \\
23\end{array}$ & $\begin{array}{c}3,58 \\
\pm \\
0,14 \\
64\end{array}$ & $\begin{array}{c}3,15 \\
\pm \\
0,12 \\
30\end{array}$ & $\begin{array}{c}3,44 \\
\pm \\
0,11 \\
13\end{array}$ & $\begin{array}{c}3,44 \\
\pm \\
0,11 \\
08\end{array}$ & $\begin{array}{c}95,40 \\
\pm \\
2,148 \\
0\end{array}$ & $\begin{array}{c}37,11 \\
\pm \\
2,418 \\
4\end{array}$ & $\begin{array}{c}21,32 \\
\pm \\
2,103 \\
9\end{array}$ & $\begin{array}{c}3,44 \\
\pm \\
0,11 \\
10\end{array}$ & $\begin{array}{c}3,44 \\
\pm \\
0,11 \\
10\end{array}$ & $\begin{array}{c}0,99 \\
\pm \\
0,03 \\
39\end{array}$ & $\begin{array}{c}28,41 \\
\pm \\
1,560 \\
6\end{array}$ \\
\hline 14 & $\begin{array}{c}3,25 \\
\pm \\
0,21 \\
14\end{array}$ & $\begin{array}{c}3,19 \\
\pm \\
0,28 \\
04\end{array}$ & $\begin{array}{c}3,09 \\
\pm \\
0,11 \\
04\end{array}$ & $\begin{array}{c}3,18 \\
\pm \\
0,14 \\
96\end{array}$ & $\begin{array}{c}3,17 \\
\pm \\
0,14 \\
74\end{array}$ & $\begin{array}{c}97,71 \\
\pm \\
3,696 \\
1\end{array}$ & $\begin{array}{c}31,70 \\
\pm \\
2,906 \\
7\end{array}$ & $\begin{array}{c}16,83 \\
\pm \\
2,283 \\
3\end{array}$ & $\begin{array}{c}3,18 \\
\pm \\
0,14 \\
85\end{array}$ & $\begin{array}{c}3,18 \\
\pm \\
0,14 \\
85\end{array}$ & $\begin{array}{c}0,98 \\
\pm \\
0,07 \\
58\end{array}$ & $\begin{array}{c}25,75 \\
\pm \\
1,469 \\
5\end{array}$ \\
\hline 15 & $\begin{array}{c}3,44 \\
\pm \\
0,18 \\
60\end{array}$ & $\begin{array}{c}3,28 \\
\pm \\
0,21 \\
85\end{array}$ & $\begin{array}{c}2,94 \\
\pm \\
0,21 \\
75\end{array}$ & $\begin{array}{c}3,22 \\
\pm \\
0,16 \\
39\end{array}$ & $\begin{array}{c}3,21 \\
\pm \\
0,16 \\
43\end{array}$ & $\begin{array}{c}93,44 \\
\pm \\
3,407 \\
5\end{array}$ & $\begin{array}{c}32,39 \\
\pm \\
3,348 \\
5\end{array}$ & $\begin{array}{c}5,40 \pm \\
0,558 \\
1\end{array}$ & $\begin{array}{c}3,21 \\
\pm \\
0,16 \\
41\end{array}$ & $\begin{array}{c}3,21 \\
\pm \\
0,16 \\
40\end{array}$ & $\begin{array}{c}0,95 \\
\pm \\
0,05 \\
50\end{array}$ & $\begin{array}{c}21,63 \\
\pm \\
1,239 \\
5\end{array}$ \\
\hline 17 & $\begin{array}{c}5,18 \\
\pm \\
0,24 \\
87\end{array}$ & $\begin{array}{c}5,08 \\
\pm \\
0,26 \\
87\end{array}$ & $\begin{array}{c}3,90 \\
\pm \\
0,15 \\
56\end{array}$ & $\begin{array}{c}4,72 \\
\pm \\
0,12 \\
39\end{array}$ & $\begin{array}{c}4,68 \\
\pm \\
0,11 \\
91\end{array}$ & $\begin{array}{c}90,44 \\
\pm \\
2,928 \\
3\end{array}$ & $\begin{array}{c}68,77 \\
\pm \\
3,497 \\
9\end{array}$ & $\begin{array}{c}53,67 \\
\pm \\
4,091 \\
2\end{array}$ & $\begin{array}{c}4,70 \\
\pm \\
0,12 \\
16\end{array}$ & $\begin{array}{c}4,70 \\
\pm \\
0,12 \\
14\end{array}$ & $\begin{array}{c}0,98 \\
\pm \\
0,07 \\
48\end{array}$ & $\begin{array}{c}79,36 \\
\pm \\
2,025 \\
4\end{array}$ \\
\hline 18 & $\begin{array}{c}5,04 \\
\pm \\
0,46 \\
82\end{array}$ & $\begin{array}{c}4,73 \\
\pm \\
0,56 \\
16\end{array}$ & $\begin{array}{c}3,77 \\
\pm \\
0,60 \\
29\end{array}$ & $\begin{array}{c}4,51 \\
\pm \\
0,48 \\
24\end{array}$ & $\begin{array}{c}4,47 \\
\pm \\
0,50 \\
00\end{array}$ & $\begin{array}{c}88,67 \\
\pm \\
5,186 \\
9\end{array}$ & $\begin{array}{c}63,40 \\
\pm \\
13,65 \\
31\end{array}$ & $\begin{array}{c}48,23 \\
\pm \\
15,05 \\
84\end{array}$ & $\begin{array}{c}4,49 \\
\pm \\
0,49 \\
05\end{array}$ & $\begin{array}{c}4,49 \\
\pm \\
0,49 \\
09\end{array}$ & $\begin{array}{c}0,94 \\
\pm \\
0,08 \\
17\end{array}$ & $\begin{array}{c}58,03 \\
\pm \\
5,841 \\
7\end{array}$ \\
\hline 19 & $\begin{array}{c}2,11 \\
\pm\end{array}$ & $\begin{array}{c}2,15 \\
\pm\end{array}$ & $\begin{array}{c}2,00 \\
\pm\end{array}$ & $\begin{array}{c}2,09 \\
\pm\end{array}$ & $\begin{array}{c}2,08 \\
\pm\end{array}$ & $\begin{array}{c}98,83 \\
\pm\end{array}$ & $\begin{array}{c}13,90 \\
\pm\end{array}$ & $\begin{array}{l}5,02 \pm \\
2,225\end{array}$ & $\begin{array}{c}2,08 \\
\pm\end{array}$ & $\begin{array}{c}2,08 \\
\pm\end{array}$ & $\begin{array}{c}1,02 \\
\pm\end{array}$ & $\begin{array}{l}7,06 \pm \\
0,427\end{array}$ \\
\hline
\end{tabular}




\begin{tabular}{|c|c|c|c|c|c|c|c|c|c|c|c|c|}
\hline & $\begin{array}{c}0,36 \\
30\end{array}$ & $\begin{array}{c}0,36 \\
28\end{array}$ & $\begin{array}{c}0,27 \\
86\end{array}$ & $\begin{array}{c}0,31 \\
80\end{array}$ & $\begin{array}{c}0,31 \\
67\end{array}$ & $\begin{array}{c}4,234 \\
7\end{array}$ & $\begin{array}{c}4,180 \\
0\end{array}$ & 8 & $\begin{array}{c}0,31 \\
73\end{array}$ & $\begin{array}{c}0,31 \\
73\end{array}$ & $\begin{array}{c}0,08 \\
84\end{array}$ & 9 \\
\hline 20 & $\begin{array}{c}4,59 \\
\pm \\
0,48 \\
65\end{array}$ & $\begin{array}{c}4,84 \\
\pm \\
0,82 \\
15\end{array}$ & $\begin{array}{c}3,64 \\
\pm \\
0,31 \\
66\end{array}$ & $\begin{array}{c}4,35 \\
\pm \\
0,40 \\
73\end{array}$ & $\begin{array}{c}4,31 \\
\pm \\
0,38 \\
52\end{array}$ & $\begin{array}{c}94,15 \\
\pm \\
7,058 \\
6\end{array}$ & $\begin{array}{c}58,65 \\
\pm \\
10,80 \\
78\end{array}$ & $\begin{array}{c}42,70 \\
\pm \\
12,15 \\
85\end{array}$ & $\begin{array}{c}4,33 \\
\pm \\
0,39 \\
64\end{array}$ & $\begin{array}{c}4,33 \\
\pm \\
0,39 \\
61\end{array}$ & $\begin{array}{c}1,06 \\
\pm \\
0,16 \\
82\end{array}$ & $\begin{array}{c}49,56 \\
\pm \\
1,910 \\
6\end{array}$ \\
\hline 22 & $\begin{array}{c}5,06 \\
\pm \\
0,65 \\
63\end{array}$ & $\begin{array}{c}5,17 \\
\pm \\
0,48 \\
30\end{array}$ & $\begin{array}{c}4,06 \\
\pm \\
0,40 \\
22\end{array}$ & $\begin{array}{c}4,76 \\
\pm \\
0,38 \\
17\end{array}$ & $\begin{array}{c}4,72 \\
\pm \\
0,37 \\
52\end{array}$ & $\begin{array}{c}93,97 \\
\pm \\
5,434 \\
8\end{array}$ & $\begin{array}{c}70,50 \\
\pm \\
11,04 \\
45\end{array}$ & $\begin{array}{c}56,12 \\
\pm \\
13,01 \\
91\end{array}$ & $\begin{array}{c}4,74 \\
\pm \\
0,37 \\
83\end{array}$ & $\begin{array}{c}4,74 \\
\pm \\
0,37 \\
83\end{array}$ & $\begin{array}{c}1,03 \\
\pm \\
0,13 \\
94\end{array}$ & $\begin{array}{c}60,33 \\
\pm \\
5,040 \\
7\end{array}$ \\
\hline 23 & $\begin{array}{c}7,14 \\
\pm \\
0,25 \\
16\end{array}$ & $\begin{array}{c}6,12 \\
\pm \\
0,64 \\
31\end{array}$ & $\begin{array}{c}6,57 \\
\pm \\
0,31 \\
42\end{array}$ & $\begin{array}{c}6,61 \\
\pm \\
0,33 \\
08\end{array}$ & $\begin{array}{c}6,59 \\
\pm \\
0,33 \\
70\end{array}$ & $\begin{array}{c}92,21 \\
\pm \\
2,342 \\
2\end{array}$ & $\begin{array}{c}136,7 \\
0 \pm \\
14,06 \\
86\end{array}$ & $\begin{array}{c}150,8 \\
2 \pm \\
23,41 \\
44\end{array}$ & $\begin{array}{c}6,60 \\
\pm \\
0,33 \\
38\end{array}$ & $\begin{array}{c}6,60 \\
\pm \\
0,33 \\
39\end{array}$ & $\begin{array}{c}0,86 \\
\pm \\
0,07 \\
24\end{array}$ & $\begin{array}{c}189.2 \\
6 \pm \\
9,698 \\
9\end{array}$ \\
\hline 26 & $\begin{array}{c}4,78 \\
\pm \\
0,21 \\
09\end{array}$ & $\begin{array}{c}4,57 \\
\pm \\
0,25 \\
33\end{array}$ & $\begin{array}{c}3,98 \\
\pm \\
0,22 \\
93\end{array}$ & $\begin{array}{c}4,44 \\
\pm \\
0,16 \\
12\end{array}$ & $\begin{array}{c}4,42 \\
\pm \\
0,16 \\
27\end{array}$ & $\begin{array}{c}92,66 \\
\pm \\
1,399 \\
9\end{array}$ & $\begin{array}{c}61,58 \\
\pm \\
4,500 \\
3\end{array}$ & $\begin{array}{c}45,52 \\
\pm \\
4,962 \\
0\end{array}$ & $\begin{array}{c}4,43 \\
\pm \\
0,16 \\
18\end{array}$ & $\begin{array}{c}4,43 \\
\pm \\
0,16 \\
19\end{array}$ & $\begin{array}{c}0,96 \\
\pm \\
0,05 \\
81\end{array}$ & $\begin{array}{c}60,71 \\
\pm \\
1,758 \\
1\end{array}$ \\
\hline 27 & $\begin{array}{c}5,23 \\
\pm \\
0,17 \\
63\end{array}$ & $\begin{array}{c}4,84 \\
\pm \\
0,44 \\
90\end{array}$ & $\begin{array}{c}4,26 \\
\pm \\
0,36 \\
81\end{array}$ & $\begin{array}{c}4,78 \\
\pm \\
0,21 \\
89\end{array}$ & $\begin{array}{c}4,75 \\
\pm \\
0,22 \\
50\end{array}$ & $\begin{array}{c}90,79 \\
\pm \\
2,852 \\
2\end{array}$ & $\begin{array}{c}70,95 \\
\pm \\
6,778 \\
2\end{array}$ & $\begin{array}{c}56,54 \\
\pm \\
8,136 \\
6\end{array}$ & $\begin{array}{c}4,77 \\
\pm \\
0,22 \\
16\end{array}$ & $\begin{array}{c}4,76 \\
\pm \\
0,22 \\
17\end{array}$ & $\begin{array}{c}0,92 \\
\pm \\
0,07 \\
62\end{array}$ & $\begin{array}{c}68,19 \\
\pm \\
5,353 \\
1\end{array}$ \\
\hline 29 & $\begin{array}{c}5,42 \\
\pm \\
0,26 \\
29\end{array}$ & $\begin{array}{c}4,72 \\
\pm \\
0,41 \\
19\end{array}$ & $\begin{array}{c}4,11 \\
\pm \\
0,57 \\
32\end{array}$ & $\begin{array}{c}4,75 \\
\pm \\
0,20 \\
13\end{array}$ & $\begin{array}{c}4,70 \\
\pm \\
0,21 \\
55\end{array}$ & $\begin{array}{c}86,78 \\
\pm \\
3,700 \\
3\end{array}$ & $\begin{array}{c}69,58 \\
\pm \\
6,337 \\
1\end{array}$ & $\begin{array}{c}54,73 \\
\pm \\
7,434 \\
3\end{array}$ & $\begin{array}{c}4,73 \\
\pm \\
0,20 \\
67\end{array}$ & $\begin{array}{c}4,73 \\
\pm \\
0,20 \\
73\end{array}$ & $\begin{array}{c}0,87 \\
\pm \\
0,06 \\
60\end{array}$ & $\begin{array}{c}72,20 \\
\pm \\
5,451 \\
5\end{array}$ \\
\hline 32 & $\begin{array}{c}4,39 \\
\pm \\
0,16 \\
50\end{array}$ & $\begin{array}{c}4,88 \\
\pm \\
0,27 \\
04\end{array}$ & $\begin{array}{c}3,80 \\
\pm \\
0,29 \\
30\end{array}$ & $\begin{array}{c}4,36 \\
\pm \\
0,19 \\
26\end{array}$ & $\begin{array}{c}4,33 \\
\pm \\
0,19 \\
71\end{array}$ & $\begin{array}{c}98,56 \\
\pm \\
2,601 \\
3\end{array}$ & $\begin{array}{c}59,03 \\
\pm \\
5,389 \\
0\end{array}$ & $\begin{array}{c}42,76 \\
\pm \\
5,871 \\
4\end{array}$ & $\begin{array}{c}4,34 \\
\pm \\
0,19 \\
45\end{array}$ & $\begin{array}{c}4,34 \\
\pm \\
0,19 \\
47\end{array}$ & $\begin{array}{c}1,11 \\
\pm \\
0,05 \\
80\end{array}$ & $\begin{array}{c}63,21 \\
\pm \\
2,478 \\
1\end{array}$ \\
\hline 33 & $\begin{array}{c}3,90 \\
\pm \\
0,18 \\
47\end{array}$ & $\begin{array}{c}3,87 \\
\pm \\
0,19 \\
28\end{array}$ & $\begin{array}{c}3,46 \\
\pm \\
0,21 \\
37\end{array}$ & $\begin{array}{c}3,74 \\
\pm \\
0,17 \\
64\end{array}$ & $\begin{array}{c}3,74 \\
\pm \\
0,17 \\
79\end{array}$ & $\begin{array}{c}95,88 \\
\pm \\
2,016 \\
6\end{array}$ & $\begin{array}{c}43,98 \\
\pm \\
4,147 \\
5\end{array}$ & $\begin{array}{c}27,51 \\
\pm \\
3,854 \\
6\end{array}$ & $\begin{array}{c}3,74 \\
\pm \\
0,17 \\
71\end{array}$ & $\begin{array}{c}3,74 \\
\pm \\
0,17 \\
71\end{array}$ & $\begin{array}{c}0,99 \\
\pm \\
0,04 \\
41\end{array}$ & $\begin{array}{c}36,32 \\
\pm \\
2,089 \\
6\end{array}$ \\
\hline 34 & $\begin{array}{c}6,67 \\
\pm \\
0,65 \\
47\end{array}$ & $\begin{array}{c}5,85 \\
\pm \\
0,63 \\
70\end{array}$ & $\begin{array}{c}6,05 \\
\pm \\
0,61 \\
69\end{array}$ & $\begin{array}{c}6,19 \\
\pm \\
0,39 \\
15\end{array}$ & $\begin{array}{c}6,16 \\
\pm \\
0,37 \\
70\end{array}$ & $\begin{array}{c}92,76 \\
\pm \\
4,842 \\
6\end{array}$ & $\begin{array}{c}119,5 \\
7 \pm \\
14,26 \\
90\end{array}$ & $\begin{array}{c}123,5 \\
4 \pm \\
21,54 \\
67\end{array}$ & $\begin{array}{c}6,17 \\
\pm \\
0,38 \\
45\end{array}$ & $\begin{array}{c}6,17 \\
\pm \\
0,38 \\
42\end{array}$ & $\begin{array}{c}0,89 \\
\pm \\
0,13 \\
52\end{array}$ & $\begin{array}{c}177,4 \\
0 \pm \\
10,29 \\
07\end{array}$ \\
\hline 35 & $\begin{array}{c}2,91 \\
\pm \\
0,17 \\
59\end{array}$ & $\begin{array}{c}2,92 \\
\pm \\
0,34 \\
99\end{array}$ & $\begin{array}{c}2,45 \\
\pm \\
0,31 \\
12\end{array}$ & $\begin{array}{c}2,76 \\
\pm \\
0,19 \\
77\end{array}$ & $\begin{array}{c}2,74 \\
\pm \\
0,20 \\
03\end{array}$ & $\begin{array}{c}94,44 \\
\pm \\
5,529 \\
0\end{array}$ & $\begin{array}{c}23,75 \\
\pm \\
3,551 \\
7\end{array}$ & $\begin{array}{c}10,96 \\
\pm \\
2,524 \\
1\end{array}$ & $\begin{array}{c}2,75 \\
\pm \\
0,19 \\
80\end{array}$ & $\begin{array}{c}2,75 \\
\pm \\
0,19 \\
85\end{array}$ & $\begin{array}{c}1,00 \\
\pm \\
0,10 \\
22\end{array}$ & $\begin{array}{c}19,21 \\
\pm \\
2,306 \\
1\end{array}$ \\
\hline 36 & $\begin{array}{c}3,05 \\
\pm \\
0,14 \\
97\end{array}$ & $\begin{array}{c}3,12 \\
\pm \\
0,15 \\
97\end{array}$ & $\begin{array}{c}3,01 \\
\pm \\
0,15 \\
13\end{array}$ & $\begin{array}{c}3,06 \\
\pm \\
0,11 \\
08\end{array}$ & $\begin{array}{c}3,07 \\
\pm \\
0,10 \\
88\end{array}$ & $\begin{array}{c}100,5 \\
3 \pm \\
3,536 \\
5\end{array}$ & $\begin{array}{c}19,27 \\
\pm \\
0,683 \\
5\end{array}$ & $\begin{array}{c}15,04 \\
\pm \\
1,632 \\
6\end{array}$ & $\begin{array}{c}3,06 \\
\pm \\
0,11 \\
11\end{array}$ & $\begin{array}{c}3,06 \\
\pm \\
0,10 \\
96\end{array}$ & $\begin{array}{c}1,02 \\
\pm \\
0,07 \\
51\end{array}$ & $\begin{array}{c}22,06 \\
\pm \\
0,639 \\
8\end{array}$ \\
\hline 37 & $\begin{array}{c}3,56 \\
\pm \\
0,21 \\
96\end{array}$ & $\begin{array}{c}3,54 \\
\pm \\
0,29 \\
57\end{array}$ & $\begin{array}{c}3,42 \\
\pm \\
0,32 \\
61\end{array}$ & $\begin{array}{c}3,51 \\
\pm \\
0,20 \\
33\end{array}$ & $\begin{array}{c}3,50 \\
\pm \\
0,20 \\
84\end{array}$ & $\begin{array}{c}98,53 \\
\pm \\
4,858 \\
1\end{array}$ & $\begin{array}{c}38,65 \\
\pm \\
4,618 \\
7\end{array}$ & $\begin{array}{c}22,70 \\
\pm \\
4,080 \\
3\end{array}$ & $\begin{array}{c}3,51 \\
\pm \\
0,20 \\
57\end{array}$ & $\begin{array}{c}3,51 \\
\pm \\
0,20 \\
58\end{array}$ & $\begin{array}{c}1,00 \\
\pm \\
0,07 \\
49\end{array}$ & $\begin{array}{c}26,73 \\
\pm \\
1,147 \\
3\end{array}$ \\
\hline
\end{tabular}




\begin{tabular}{|c|c|c|c|c|c|c|c|c|c|c|c|c|}
\hline & & & & & & & & & & & & \\
\hline 38 & $\begin{array}{c}2,95 \\
\pm \\
0,22 \\
33\end{array}$ & $\begin{array}{c}2,85 \\
\pm \\
0,35 \\
70\end{array}$ & $\begin{array}{c}2,66 \\
\pm \\
0,35 \\
03\end{array}$ & $\begin{array}{c}2,82 \\
\pm \\
0,29 \\
42\end{array}$ & $\begin{array}{c}2,82 \\
\pm \\
0,29 \\
94\end{array}$ & $\begin{array}{c}95,38 \\
\pm \\
5,073 \\
7\end{array}$ & $\begin{array}{c}25,15 \\
\pm \\
5,076 \\
8\end{array}$ & $\begin{array}{c}12,03 \\
\pm \\
3,468 \\
0\end{array}$ & $\begin{array}{c}2,82 \\
\pm \\
0,29 \\
70\end{array}$ & $\begin{array}{c}2,82 \\
\pm \\
0,29 \\
69\end{array}$ & $\begin{array}{c}0,97 \\
\pm \\
0,07 \\
93\end{array}$ & $\begin{array}{c}16,94 \\
\pm \\
1,217 \\
4\end{array}$ \\
\hline 40 & $\begin{array}{c}4,76 \\
\pm \\
0,48 \\
55\end{array}$ & $\begin{array}{c}4,48 \\
\pm \\
0,55 \\
01\end{array}$ & $\begin{array}{c}4,20 \\
\pm \\
0,39 \\
70\end{array}$ & $\begin{array}{c}4,48 \\
\pm \\
0,22 \\
55\end{array}$ & $\begin{array}{c}4,45 \\
\pm \\
0,22 \\
18\end{array}$ & $\begin{array}{c}94,37 \\
\pm \\
9,674 \\
9\end{array}$ & $\begin{array}{c}62,39 \\
\pm \\
6,353 \\
1\end{array}$ & $\begin{array}{c}46,50 \\
\pm \\
7,256 \\
0\end{array}$ & $\begin{array}{c}4,46 \\
\pm \\
0,22 \\
37\end{array}$ & $\begin{array}{c}4,46 \\
\pm \\
0,22 \\
36\end{array}$ & $\begin{array}{c}0,95 \\
\pm \\
0,17 \\
13\end{array}$ & $\begin{array}{c}61,13 \\
\pm \\
6,027 \\
2\end{array}$ \\
\hline 41 & $\begin{array}{c}5,46 \\
\pm \\
0,57 \\
25\end{array}$ & $\begin{array}{c}5,46 \\
\pm \\
0,44 \\
61\end{array}$ & $\begin{array}{c}5,09 \\
\pm \\
0,49 \\
06\end{array}$ & $\begin{array}{c}5,33 \\
\pm \\
0,40 \\
78\end{array}$ & $\begin{array}{c}5,32 \\
\pm \\
0,40 \\
80\end{array}$ & $\begin{array}{c}97,88 \\
\pm \\
5,163 \\
3\end{array}$ & $\begin{array}{c}89,50 \\
\pm \\
13,20 \\
35\end{array}$ & $\begin{array}{c}80,22 \\
\pm \\
17,16 \\
24\end{array}$ & $\begin{array}{c}5,33 \\
\pm \\
0,40 \\
79\end{array}$ & $\begin{array}{c}5,33 \\
\pm \\
0,40 \\
79\end{array}$ & $\begin{array}{c}1,00 \\
\pm \\
0,06 \\
89\end{array}$ & $\begin{array}{c}113,1 \\
0 \pm \\
1,581 \\
1\end{array}$ \\
\hline 42 & $\begin{array}{c}4,26 \\
\pm \\
0,27 \\
31\end{array}$ & $\begin{array}{c}4,28 \\
\pm \\
0,31 \\
37\end{array}$ & $\begin{array}{c}3,74 \\
\pm \\
0,12 \\
03\end{array}$ & $\begin{array}{c}4,09 \\
\pm \\
0,17 \\
63\end{array}$ & $\begin{array}{c}4,08 \\
\pm \\
0,16 \\
85\end{array}$ & $\begin{array}{c}96,02 \\
\pm \\
3,668 \\
4\end{array}$ & $\begin{array}{c}52,39 \\
\pm \\
4,316 \\
4\end{array}$ & $\begin{array}{c}35,74 \\
\pm \\
4,405 \\
4\end{array}$ & $\begin{array}{c}4,09 \\
\pm \\
0,17 \\
24\end{array}$ & $\begin{array}{c}4,09 \\
\pm \\
0,17 \\
24\end{array}$ & $\begin{array}{c}1,01 \\
\pm \\
0,06 \\
91\end{array}$ & $\begin{array}{c}48,97 \\
\pm \\
0,945 \\
2\end{array}$ \\
\hline 43 & $\begin{array}{c}6,27 \\
\pm \\
0,46 \\
60\end{array}$ & $\begin{array}{c}5,93 \\
\pm \\
0,75 \\
36\end{array}$ & $\begin{array}{c}5,06 \\
\pm \\
0,59 \\
03\end{array}$ & $\begin{array}{c}5,75 \\
\pm \\
0,53 \\
02\end{array}$ & $\begin{array}{c}5,72 \\
\pm \\
0,53 \\
31\end{array}$ & $\begin{array}{c}91,20 \\
\pm \\
3,334 \\
2\end{array}$ & $\begin{array}{c}103,6 \\
8 \pm \\
18,24 \\
47\end{array}$ & $\begin{array}{c}100,3 \\
4 \pm \\
25,02 \\
39\end{array}$ & $\begin{array}{c}5,74 \\
\pm \\
0,53 \\
17\end{array}$ & $\begin{array}{c}5,74 \\
\pm \\
0,53 \\
17\end{array}$ & $\begin{array}{c}0,94 \\
\pm \\
0,07 \\
16\end{array}$ & $\begin{array}{c}132,4 \\
4 \pm \\
8,532 \\
4\end{array}$ \\
\hline 45 & $\begin{array}{c}3,81 \\
\pm \\
0,30 \\
11\end{array}$ & $\begin{array}{c}3,70 \\
\pm \\
0,28 \\
68\end{array}$ & $\begin{array}{c}3,42 \\
\pm \\
0,28 \\
45\end{array}$ & $\begin{array}{c}3,64 \\
\pm \\
0,27 \\
10\end{array}$ & $\begin{array}{c}3,64 \\
\pm \\
0,27 \\
20\end{array}$ & $\begin{array}{c}95,60 \\
\pm \\
1,987 \\
5\end{array}$ & $\begin{array}{c}41,78 \\
\pm \\
6,217 \\
3\end{array}$ & $\begin{array}{c}25,58 \\
\pm \\
5,680 \\
8\end{array}$ & $\begin{array}{c}3,64 \\
\pm \\
0,27 \\
15\end{array}$ & $\begin{array}{c}3,64 \\
\pm \\
0,27 \\
15\end{array}$ & $\begin{array}{c}0,97 \\
\pm \\
0,03 \\
96\end{array}$ & $\begin{array}{c}36,15 \\
\pm \\
1,787 \\
7\end{array}$ \\
\hline 46 & $\begin{array}{c}5,27 \\
\pm \\
0,41 \\
87\end{array}$ & $\begin{array}{c}5,80 \\
\pm \\
0,43 \\
15\end{array}$ & $\begin{array}{c}3,57 \\
\pm \\
0,23 \\
29\end{array}$ & $\begin{array}{c}4,88 \\
\pm \\
0,33 \\
22\end{array}$ & $\begin{array}{c}4,78 \\
\pm \\
0,31 \\
85\end{array}$ & $\begin{array}{c}90,74 \\
\pm \\
3,416 \\
9\end{array}$ & $\begin{array}{c}71,95 \\
\pm \\
9,152 \\
6\end{array}$ & $\begin{array}{c}57,68 \\
\pm \\
10,49 \\
79\end{array}$ & $\begin{array}{c}4,83 \\
\pm \\
0,32 \\
59\end{array}$ & $\begin{array}{c}4,83 \\
\pm \\
0,32 \\
54\end{array}$ & $\begin{array}{c}1,10 \\
\pm \\
0,05 \\
18\end{array}$ & $\begin{array}{c}60,82 \\
\pm \\
\mathbf{1 , 1 5 8} \\
\mathbf{3}\end{array}$ \\
\hline 47 & $\begin{array}{c}3,88 \\
\pm \\
0,20 \\
83\end{array}$ & $\begin{array}{c}3,70 \\
\pm \\
0,17 \\
67\end{array}$ & $\begin{array}{c}3,38 \\
\pm \\
0,22 \\
62\end{array}$ & $\begin{array}{c}3,65 \\
\pm \\
0,18 \\
34\end{array}$ & $\begin{array}{c}3,65 \\
\pm \\
0,18 \\
41\end{array}$ & $\begin{array}{c}93,98 \\
\pm \\
1,612 \\
0\end{array}$ & $\begin{array}{c}41,85 \\
\pm \\
4,225 \\
1\end{array}$ & $\begin{array}{c}25,54 \\
\pm \\
3,865 \\
0\end{array}$ & $\begin{array}{c}3,65 \\
\pm \\
0,18 \\
37\end{array}$ & $\begin{array}{c}3,65 \\
\pm \\
0,18 \\
37\end{array}$ & $\begin{array}{c}0,95 \\
\pm \\
0,03 \\
12\end{array}$ & $\begin{array}{c}31,04 \\
\pm \\
3,140 \\
0\end{array}$ \\
\hline 49 & $\begin{array}{c}4,71 \\
\pm \\
0,36 \\
72\end{array}$ & $\begin{array}{c}4,13 \\
\pm \\
0,39 \\
42\end{array}$ & $\begin{array}{c}3,84 \\
\pm \\
0,26 \\
58\end{array}$ & $\begin{array}{c}4,23 \\
\pm \\
0,23 \\
07\end{array}$ & $\begin{array}{c}4,20 \\
\pm \\
0,23 \\
19\end{array}$ & $\begin{array}{c}89,41 \\
\pm \\
4,867 \\
5\end{array}$ & $\begin{array}{c}55,65 \\
\pm \\
6,099 \\
6\end{array}$ & $\begin{array}{c}39,20 \\
\pm \\
6,394 \\
0\end{array}$ & $\begin{array}{c}4,21 \\
\pm \\
2,28 \\
97\end{array}$ & $\begin{array}{c}4,21 \\
\pm \\
0,23 \\
11\end{array}$ & $\begin{array}{c}0,88 \\
\pm \\
0,10 \\
88\end{array}$ & $\begin{array}{c}55,97 \\
\pm \\
3,568 \\
1\end{array}$ \\
\hline 51 & $\begin{array}{c}3,92 \\
\pm \\
0,17 \\
94\end{array}$ & $\begin{array}{c}3,99 \\
\pm \\
0,33 \\
20\end{array}$ & $\begin{array}{c}2,56 \\
\pm \\
0,15 \\
33\end{array}$ & $\begin{array}{c}3,49 \\
\pm \\
0,11 \\
40\end{array}$ & $\begin{array}{c}3,42 \\
\pm \\
0,10 \\
02\end{array}$ & $\begin{array}{c}87,34 \\
\pm \\
4,135 \\
4\end{array}$ & $\begin{array}{c}36,68 \\
\pm \\
2,169 \\
1\end{array}$ & $\begin{array}{c}20,91 \\
\pm \\
1,869 \\
1\end{array}$ & $\begin{array}{c}3,46 \\
\pm \\
0,10 \\
74\end{array}$ & $\begin{array}{c}3,46 \\
\pm \\
0,08 \\
00\end{array}$ & $\begin{array}{c}1,02 \\
\pm \\
0,09 \\
26\end{array}$ & $\begin{array}{c}25,28 \\
\pm \\
0,421 \\
2\end{array}$ \\
\hline 55 & $\begin{array}{c}5,89 \\
\pm \\
0,50 \\
13\end{array}$ & $\begin{array}{c}5,38 \\
\pm \\
0,96 \\
55\end{array}$ & $\begin{array}{c}5,25 \\
\pm \\
0,36 \\
86\end{array}$ & $\begin{array}{c}5,50 \\
\pm \\
0,42 \\
48\end{array}$ & $\begin{array}{c}5,48 \\
\pm \\
0,42 \\
17\end{array}$ & $\begin{array}{c}93,19 \\
\pm \\
4,543 \\
8\end{array}$ & $\begin{array}{c}94,68 \\
\pm \\
14,70 \\
06\end{array}$ & $\begin{array}{c}87,33 \\
\pm \\
20,51 \\
39\end{array}$ & $\begin{array}{c}5,49 \\
\pm \\
0,42 \\
28\end{array}$ & $\begin{array}{c}5,49 \\
\pm \\
0,42 \\
30\end{array}$ & $\begin{array}{c}0,91 \\
\pm \\
0,14 \\
53\end{array}$ & $\begin{array}{c}111,5 \\
5 \pm \\
6,623 \\
5\end{array}$ \\
\hline 56 & $\begin{array}{c}4,46 \\
\pm \\
0,55 \\
43\end{array}$ & $\begin{array}{c}5,19 \\
\pm \\
0,61 \\
30\end{array}$ & $\begin{array}{c}3,58 \\
\pm \\
0,35 \\
39\end{array}$ & $\begin{array}{c}4,41 \\
\pm \\
0,31 \\
50\end{array}$ & $\begin{array}{c}4,34 \\
\pm \\
0,31 \\
33\end{array}$ & $\begin{array}{c}98,28 \\
\pm \\
9,170 \\
2\end{array}$ & $\begin{array}{c}59,56 \\
\pm \\
8,326 \\
9\end{array}$ & $\begin{array}{c}43,51 \\
\pm \\
8,855 \\
0\end{array}$ & $\begin{array}{c}4,38 \\
\pm \\
0,31 \\
37\end{array}$ & $\begin{array}{c}4,38 \\
\pm \\
0,31 \\
38\end{array}$ & $\begin{array}{c}1,18 \\
\pm \\
0,20 \\
27\end{array}$ & $\begin{array}{c}61,94 \\
\pm \\
2,490 \\
8\end{array}$ \\
\hline 57 & 4,93 & 5,29 & 4,10 & 4,78 & 4,74 & 96,43 & 70,84 & 56,33 & 4,76 & 4,76 & 1,08 & 80,67 \\
\hline
\end{tabular}




\begin{tabular}{|c|c|c|c|c|c|c|c|c|c|c|c|c|}
\hline & $\begin{array}{c} \pm \\
0,39 \\
23\end{array}$ & $\begin{array}{c} \pm \\
0,43 \\
64\end{array}$ & $\begin{array}{c} \pm \\
0,33 \\
04\end{array}$ & $\begin{array}{c} \pm \\
0,27 \\
91\end{array}$ & $\begin{array}{c} \pm \\
0,27 \\
72\end{array}$ & $\begin{array}{c} \pm \\
5,269 \\
3\end{array}$ & $\begin{array}{c} \pm \\
8,390 \\
5\end{array}$ & $\begin{array}{c} \pm \\
10,14 \\
28\end{array}$ & $\begin{array}{c} \pm \\
0,27 \\
79\end{array}$ & $\begin{array}{c} \pm \\
0,27 \\
79\end{array}$ & $\begin{array}{c} \pm \\
0,11 \\
20\end{array}$ & $\begin{array}{c} \pm \\
5,025\end{array}$ \\
\hline 59 & $\begin{array}{c}3,78 \\
\pm \\
0,19 \\
21\end{array}$ & $\begin{array}{c}3,74 \\
\pm \\
0,23 \\
30\end{array}$ & $\begin{array}{c}3,31 \\
\pm \\
0,31 \\
58\end{array}$ & $\begin{array}{c}3,61 \\
\pm \\
0,22 \\
94\end{array}$ & $\begin{array}{c}3,60 \\
\pm \\
0,23 \\
38\end{array}$ & $\begin{array}{c}95,22 \\
\pm \\
2,332 \\
0\end{array}$ & $\begin{array}{c}40,91 \\
\pm \\
5,226 \\
7\end{array}$ & $\begin{array}{c}24,74 \\
\pm \\
4,661 \\
9\end{array}$ & $\begin{array}{c}3,61 \\
\pm \\
0,23 \\
15\end{array}$ & $\begin{array}{c}3,61 \\
\pm \\
0,23 \\
15\end{array}$ & $\begin{array}{c}0,99 \\
\pm \\
0,01 \\
49\end{array}$ & $\begin{array}{c}36,78 \\
\pm \\
2,057 \\
8\end{array}$ \\
\hline 63 & $\begin{array}{c}5,33 \\
\pm \\
0,55 \\
18\end{array}$ & $\begin{array}{c}5,26 \\
\pm \\
0,42 \\
11\end{array}$ & $\begin{array}{c}4,18 \\
\pm \\
0,17 \\
52\end{array}$ & $\begin{array}{c}4,92 \\
\pm \\
0,34 \\
62\end{array}$ & $\begin{array}{c}4,89 \\
\pm \\
0,32 \\
36\end{array}$ & $\begin{array}{c}92,11 \\
\pm \\
3,512 \\
0\end{array}$ & $\begin{array}{c}75,37 \\
\pm \\
10,24 \\
70\end{array}$ & $\begin{array}{c}61,91 \\
\pm \\
12,94 \\
99\end{array}$ & $\begin{array}{c}4,91 \\
\pm \\
0,33 \\
58\end{array}$ & $\begin{array}{c}4,91 \\
\pm \\
0,33 \\
52\end{array}$ & $\begin{array}{c}0,99 \\
\pm \\
0,03 \\
39\end{array}$ & $\begin{array}{c}82,05 \\
\pm \\
3,971 \\
8\end{array}$ \\
\hline 64 & $\begin{array}{c}3,32 \\
\pm \\
0,24 \\
18\end{array}$ & $\begin{array}{c}3,36 \\
\pm \\
0,35 \\
45\end{array}$ & $\begin{array}{c}3,18 \\
\pm \\
0,20 \\
13\end{array}$ & $\begin{array}{c}3,29 \\
\pm \\
0,24 \\
22\end{array}$ & $\begin{array}{c}3,28 \\
\pm \\
0,23 \\
89\end{array}$ & $\begin{array}{c}98,94 \\
\pm \\
2,020 \\
2\end{array}$ & $\begin{array}{c}34,02 \\
\pm \\
5,160 \\
4\end{array}$ & $\begin{array}{c}18,80 \\
\pm \\
4,463 \\
8\end{array}$ & $\begin{array}{c}3,28 \\
\pm \\
0,24 \\
05\end{array}$ & $\begin{array}{c}3,28 \\
\pm \\
0,24 \\
05\end{array}$ & $\begin{array}{c}1,01 \\
\pm \\
0,06 \\
33\end{array}$ & $\begin{array}{c}25,62 \\
\pm \\
0,776 \\
2\end{array}$ \\
\hline 65 & $\begin{array}{c}3,29 \\
\pm \\
0,25 \\
17\end{array}$ & $\begin{array}{c}3,20 \\
\pm \\
0,25 \\
41\end{array}$ & $\begin{array}{c}2,76 \\
\pm \\
0,42 \\
29\end{array}$ & $\begin{array}{c}3,08 \\
\pm \\
0,21 \\
73\end{array}$ & $\begin{array}{c}3,06 \\
\pm \\
0,22 \\
98\end{array}$ & $\begin{array}{c}93,12 \\
\pm \\
4,480 \\
1\end{array}$ & $\begin{array}{c}29,63 \\
\pm \\
4,466 \\
7\end{array}$ & $\begin{array}{c}15,28 \\
\pm \\
3,467 \\
5\end{array}$ & $\begin{array}{c}3,07 \\
\pm \\
0,22 \\
26\end{array}$ & $\begin{array}{c}3,07 \\
\pm \\
0,22 \\
30\end{array}$ & $\begin{array}{c}0,97 \\
\pm \\
0,09 \\
01\end{array}$ & $\begin{array}{c}22,94 \\
\pm \\
0,482 \\
8\end{array}$ \\
\hline 70 & $\begin{array}{c}, 84 \\
\pm \\
0,35 \\
78\end{array}$ & $\begin{array}{c}4,77 \\
\pm \\
0,47 \\
10\end{array}$ & $\begin{array}{c}4,10 \\
\pm \\
0,24 \\
56\end{array}$ & $\begin{array}{c}4,57 \\
\pm \\
0,23 \\
46\end{array}$ & $\begin{array}{c}4,55 \\
\pm \\
0,22 \\
28\end{array}$ & $\begin{array}{c}94,17 \\
\pm \\
3,139 \\
7\end{array}$ & $\begin{array}{c}65,13 \\
\pm \\
6,425 \\
1\end{array}$ & $\begin{array}{c}49,58 \\
\pm \\
7,385 \\
7\end{array}$ & $\begin{array}{c}4,56 \\
\pm \\
0,22 \\
87\end{array}$ & $\begin{array}{c}4,56 \\
\pm \\
0,22 \\
86\end{array}$ & $\begin{array}{c}0,99 \\
\pm \\
0,08 \\
82\end{array}$ & $\begin{array}{c}68,26 \\
\pm \\
1,832 \\
1\end{array}$ \\
\hline 71 & $\begin{array}{c}3,41 \\
\pm \\
0,23 \\
97\end{array}$ & $\begin{array}{c}3,34 \\
\pm \\
0,27 \\
33\end{array}$ & $\begin{array}{c}3,21 \\
\pm \\
0,24 \\
60\end{array}$ & $\begin{array}{c}3,32 \\
\pm \\
0,24 \\
40\end{array}$ & $\begin{array}{c}3,32 \\
\pm \\
0,24 \\
44\end{array}$ & $\begin{array}{c}97,19 \\
\pm \\
1,365 \\
3\end{array}$ & $\begin{array}{c}34,70 \\
\pm \\
5,051 \\
0\end{array}$ & $\begin{array}{c}19,36 \\
\pm \\
4,172 \\
4\end{array}$ & $\begin{array}{c}3,32 \\
\pm \\
0,24 \\
42\end{array}$ & $\begin{array}{c}3,32 \\
\pm \\
0,24 \\
42\end{array}$ & $\begin{array}{c}0,98 \\
\pm \\
0,04 \\
01\end{array}$ & $\begin{array}{c}26,83 \\
\pm \\
1,925 \\
6\end{array}$ \\
\hline 72 & $\begin{array}{c}4,41 \\
\pm \\
0,36 \\
88\end{array}$ & $\begin{array}{c}4,42 \\
\pm \\
0,38 \\
54\end{array}$ & $\begin{array}{c}3,71 \\
\pm \\
0,35 \\
95\end{array}$ & $\begin{array}{c}4,18 \\
\pm \\
0,34 \\
56\end{array}$ & $\begin{array}{c}4,16 \\
\pm \\
0,34 \\
56\end{array}$ & $\begin{array}{c}94,47 \\
\pm \\
1,873 \\
8\end{array}$ & $\begin{array}{c}54,71 \\
\pm \\
8,863 \\
0\end{array}$ & $\begin{array}{c}38,40 \\
\pm \\
9,120 \\
9\end{array}$ & $\begin{array}{c}4,17 \\
\pm \\
0,34 \\
55\end{array}$ & $\begin{array}{c}4,17 \\
\pm \\
0,34 \\
56\end{array}$ & $\begin{array}{c}1,00 \\
\pm \\
0,02 \\
88\end{array}$ & $\begin{array}{c}51,01 \\
\pm \\
1,938 \\
7\end{array}$ \\
\hline 74 & $\begin{array}{c}4,65 \\
\pm \\
0,47 \\
26\end{array}$ & $\begin{array}{c}4,53 \\
\pm \\
0,41 \\
75\end{array}$ & $\begin{array}{c}3,94 \\
\pm \\
0,33 \\
03\end{array}$ & $\begin{array}{c}4,37 \\
\pm \\
0,35 \\
56\end{array}$ & $\begin{array}{c}4,36 \\
\pm \\
0,35 \\
13\end{array}$ & $\begin{array}{c}93,98 \\
\pm \\
2,740 \\
3\end{array}$ & $\begin{array}{c}27,38 \\
\pm \\
2,207 \\
0\end{array}$ & $\begin{array}{c}44,06 \\
\pm \\
9,548 \\
3\end{array}$ & $\begin{array}{c}4,37 \\
\pm \\
0,35 \\
35\end{array}$ & $\begin{array}{c}4,37 \\
\pm \\
0,35 \\
34\end{array}$ & $\begin{array}{c}0,98 \\
\pm \\
0,07 \\
12\end{array}$ & $\begin{array}{c}54,47 \\
\pm \\
10,15 \\
58\end{array}$ \\
\hline 77 & $\begin{array}{c}4,63 \\
\pm \\
0,32 \\
61\end{array}$ & $\begin{array}{c}4,68 \\
\pm \\
0,42 \\
05\end{array}$ & $\begin{array}{c}3,92 \\
\pm \\
0,43 \\
20\end{array}$ & $\begin{array}{c}4,41 \\
\pm \\
0,32 \\
88\end{array}$ & $\begin{array}{c}4,39 \\
\pm \\
0,33 \\
21\end{array}$ & $\begin{array}{c}94,87 \\
\pm \\
3,655 \\
6\end{array}$ & $\begin{array}{c}60,86 \\
\pm \\
8,564 \\
2\end{array}$ & $\begin{array}{c}44,96 \\
\pm \\
8,853 \\
3\end{array}$ & $\begin{array}{c}4,40 \\
\pm \\
0,33 \\
02\end{array}$ & $\begin{array}{c}4,40 \\
\pm \\
0,33 \\
03\end{array}$ & $\begin{array}{c}1,01 \\
\pm \\
0,05 \\
79\end{array}$ & $\begin{array}{c}59,82 \\
\pm \\
1,654 \\
7\end{array}$ \\
\hline 79 & $\begin{array}{c}5,55 \\
\pm \\
0,41 \\
21\end{array}$ & $\begin{array}{c}5,86 \\
\pm \\
0,59 \\
29\end{array}$ & $\begin{array}{c}, 32 \\
\pm \\
0,33 \\
15\end{array}$ & $\begin{array}{c}5,24 \\
\pm \\
0,35 \\
88\end{array}$ & $\begin{array}{c}5,19 \\
\pm \\
0,34 \\
79\end{array}$ & $\begin{array}{c}93,63 \\
\pm \\
4,279 \\
3\end{array}$ & $\begin{array}{c}85,05 \\
\pm \\
11,52 \\
70\end{array}$ & $\begin{array}{c}74,20 \\
\pm \\
15,26 \\
17\end{array}$ & $\begin{array}{c}5,22 \\
\pm \\
0,35 \\
31\end{array}$ & $\begin{array}{c}5,22 \\
\pm \\
0,35 \\
31\end{array}$ & $\begin{array}{c}1,06 \\
\pm \\
0,09 \\
36\end{array}$ & $\begin{array}{c}108,7 \\
8 \pm \\
5,484 \\
9\end{array}$ \\
\hline 80 & $\begin{array}{c}5,49 \\
\pm \\
0,25 \\
95\end{array}$ & $\begin{array}{c}5,37 \\
\pm \\
0,38 \\
16\end{array}$ & $\begin{array}{c}4,43 \\
\pm \\
0,28 \\
32\end{array}$ & $\begin{array}{c}5,10 \\
\pm \\
0,12 \\
03\end{array}$ & $\begin{array}{c}5,06 \\
\pm \\
0,12 \\
03\end{array}$ & $\begin{array}{c}92,41 \\
\pm \\
4,000 \\
5\end{array}$ & $\begin{array}{c}80,62 \\
\pm \\
3,806 \\
1\end{array}$ & $\begin{array}{c}68,12 \\
\pm \\
4,793 \\
2\end{array}$ & $\begin{array}{c}5,08 \\
\pm \\
0,12 \\
01\end{array}$ & $\begin{array}{c}5,08 \\
\pm \\
0,12 \\
01\end{array}$ & $\begin{array}{c}0,98 \\
\pm \\
0,09 \\
87\end{array}$ & $\begin{array}{c}86,05 \\
\pm \\
6,181 \\
4\end{array}$ \\
\hline 83 & $\begin{array}{c}3,99 \\
\pm\end{array}$ & $\begin{array}{c}4,17 \\
\pm\end{array}$ & $\begin{array}{c}3,54 \\
\pm\end{array}$ & $\begin{array}{c}3,90 \\
\pm\end{array}$ & $\begin{array}{c}3,88 \\
\pm\end{array}$ & $\begin{array}{c}97,25 \\
\pm\end{array}$ & $\begin{array}{c}47,54 \\
\pm\end{array}$ & $\begin{array}{c}30,98 \\
\pm\end{array}$ & $\begin{array}{c}3,89 \\
\pm\end{array}$ & $\begin{array}{c}3,89 \\
\pm\end{array}$ & $\begin{array}{c}1,04 \\
\pm\end{array}$ & $\begin{array}{c}42,10 \\
\pm\end{array}$ \\
\hline
\end{tabular}




\begin{tabular}{|c|c|c|c|c|c|c|c|c|c|c|c|c|}
\hline & $\begin{array}{c}0,17 \\
37\end{array}$ & $\begin{array}{c}0,51 \\
18\end{array}$ & $\begin{array}{c}0,31 \\
44\end{array}$ & $\begin{array}{c}0,25 \\
09\end{array}$ & $\begin{array}{c}0,24 \\
42\end{array}$ & $\begin{array}{c}4,967 \\
0\end{array}$ & $\begin{array}{c}5,870 \\
4\end{array}$ & $\begin{array}{c}5,641 \\
9\end{array}$ & $\begin{array}{c}0,24 \\
66\end{array}$ & $\begin{array}{c}0,24 \\
71\end{array}$ & $\begin{array}{c}0,12 \\
53\end{array}$ & $\begin{array}{c}2,393 \\
3\end{array}$ \\
\hline 87 & $\begin{array}{c}5,13 \\
\pm \\
0,38 \\
18\end{array}$ & $\begin{array}{c}5,03 \\
\pm \\
0,30 \\
96\end{array}$ & $\begin{array}{c}3,97 \\
\pm \\
0,43 \\
52\end{array}$ & $\begin{array}{c}4,71 \\
\pm \\
0,34 \\
60\end{array}$ & $\begin{array}{c}4,67 \\
\pm \\
0,35 \\
51\end{array}$ & $\begin{array}{c}91,15 \\
\pm \\
1,812 \\
6\end{array}$ & $\begin{array}{c}69,00 \\
\pm \\
10,65 \\
34\end{array}$ & $\begin{array}{c}54,32 \\
\pm \\
12,77 \\
33\end{array}$ & $\begin{array}{c}4,07 \\
\pm \\
0,27 \\
54\end{array}$ & $\begin{array}{c}4,48 \\
\pm \\
0,32 \\
51\end{array}$ & $\begin{array}{c}0,98 \\
\pm \\
0,04 \\
70\end{array}$ & $\begin{array}{c}84,12 \\
\pm \\
3,886 \\
2\end{array}$ \\
\hline 89 & $\begin{array}{c}4,29 \\
\pm \\
0,31 \\
21\end{array}$ & $\begin{array}{c}4,48 \\
\pm \\
0,37 \\
54\end{array}$ & $\begin{array}{c}3,36 \\
\pm \\
0,19 \\
38\end{array}$ & $\begin{array}{c}4,04 \\
\pm \\
0,24 \\
53\end{array}$ & $\begin{array}{c}4,01 \\
\pm \\
0,23 \\
67\end{array}$ & $\begin{array}{c}93,59 \\
\pm \\
3,031 \\
0\end{array}$ & $\begin{array}{c}50,56 \\
\pm \\
5,954 \\
4\end{array}$ & $\begin{array}{c}33,97 \\
\pm \\
5,973 \\
1\end{array}$ & $\begin{array}{c}, 02 \\
\pm \\
0,24 \\
13\end{array}$ & $\begin{array}{c}4,02 \\
\pm \\
0,24 \\
10\end{array}$ & $\begin{array}{c}1,05 \\
\pm \\
0,06 \\
71\end{array}$ & $\begin{array}{c}48,07 \\
\pm \\
2,789 \\
0\end{array}$ \\
\hline 90 & $\begin{array}{c}5,20 \\
\pm \\
0,46 \\
92\end{array}$ & $\begin{array}{c}5,21 \\
\pm \\
0,40 \\
18\end{array}$ & $\begin{array}{c}3,53 \\
\pm \\
0,59 \\
64\end{array}$ & $\begin{array}{c}4,65 \\
\pm \\
0,46 \\
10\end{array}$ & $\begin{array}{c}4,57 \\
\pm \\
0,49 \\
30\end{array}$ & $\begin{array}{c}87,69 \\
\pm \\
3,829 \\
7\end{array}$ & $\begin{array}{c}28,69 \\
\pm \\
3,097 \\
5\end{array}$ & $\begin{array}{c}51,33 \\
\pm \\
14,81 \\
40\end{array}$ & $\begin{array}{c}4,61 \\
\pm \\
0,47 \\
38\end{array}$ & $\begin{array}{c}4,61 \\
\pm \\
0,47 \\
58\end{array}$ & $\begin{array}{c}1,00 \\
\pm \\
0,04 \\
72\end{array}$ & $\begin{array}{c}69,05 \\
\pm\end{array}$ \\
\hline 91 & $\begin{array}{c}2,63 \\
\pm \\
0,36 \\
06\end{array}$ & $\begin{array}{c}2,44 \\
\pm \\
0,31 \\
41\end{array}$ & $\begin{array}{c}2,13 \\
\pm \\
0,36 \\
31\end{array}$ & $\begin{array}{c}2,40 \\
\pm \\
0,33 \\
70\end{array}$ & $\begin{array}{c}2,39 \\
\pm \\
0,33 \\
88\end{array}$ & $\begin{array}{c}90,68 \\
\pm \\
2,436 \\
5\end{array}$ & $\begin{array}{c}18,25 \\
\pm \\
4,946 \\
2\end{array}$ & $\begin{array}{c}7,51 \pm \\
2,914 \\
7\end{array}$ & $\begin{array}{c}2,39 \\
\pm \\
0,33 \\
78\end{array}$ & $\begin{array}{c}2,39 \\
\pm \\
0,33 \\
79\end{array}$ & $\begin{array}{c}0,93 \\
\pm \\
0,02 \\
48\end{array}$ & $\begin{array}{c}16,21 \\
\pm \\
0,212 \\
0\end{array}$ \\
\hline 93 & $\begin{array}{c}3,79 \\
\pm \\
0,17 \\
84\end{array}$ & $\begin{array}{c}4,15 \\
\pm \\
0,17 \\
14\end{array}$ & $\begin{array}{c}3,50 \\
\pm \\
0,24 \\
11\end{array}$ & $\begin{array}{c}3,81 \\
\pm \\
0,17 \\
83\end{array}$ & $\begin{array}{c}3,80 \\
\pm \\
0,18 \\
08\end{array}$ & $\begin{array}{c}100,2 \\
4 \pm \\
2,368 \\
5\end{array}$ & $\begin{array}{c}45,47 \\
\pm \\
4,341 \\
9\end{array}$ & $\begin{array}{c}28,92 \\
\pm \\
4,154 \\
9\end{array}$ & $\begin{array}{c}3,35 \\
\pm \\
0,14 \\
51\end{array}$ & $\begin{array}{c}3,65 \\
\pm \\
0,16 \\
79\end{array}$ & $\begin{array}{c}1,09 \\
\pm \\
0,04 \\
30\end{array}$ & $\begin{array}{c}42,17 \\
\pm \\
1,375 \\
1\end{array}$ \\
\hline 100 & $\begin{array}{c}3,74 \\
\pm \\
0,17 \\
08\end{array}$ & $\begin{array}{c}3,81 \\
\pm \\
0,18 \\
76\end{array}$ & $\begin{array}{c}3,28 \\
\pm \\
0,16 \\
75\end{array}$ & $\begin{array}{c}3,61 \\
\pm \\
0,14 \\
09\end{array}$ & $\begin{array}{c}3,60 \\
\pm \\
0,14 \\
16\end{array}$ & $\begin{array}{c}96,30 \\
\pm \\
1,837 \\
5\end{array}$ & $\begin{array}{c}40,69 \\
\pm \\
3,179 \\
1\end{array}$ & $\begin{array}{c}24,46 \\
\pm \\
2,842 \\
4\end{array}$ & $\begin{array}{c}3,60 \\
\pm \\
0,14 \\
13\end{array}$ & $\begin{array}{c}3,60 \\
\pm \\
0,14 \\
13\end{array}$ & $\begin{array}{c}1,02 \\
\pm \\
0,05 \\
63\end{array}$ & $\begin{array}{c}32,97 \\
\pm \\
2,611 \\
2\end{array}$ \\
\hline 102 & $\begin{array}{c}5,26 \\
\pm \\
0,39 \\
99\end{array}$ & $\begin{array}{c}4,34 \\
\pm \\
0,36 \\
35\end{array}$ & $\begin{array}{c}, 00 \\
\pm \\
0,44 \\
90\end{array}$ & $\begin{array}{c}4,53 \\
\pm \\
0,28 \\
03\end{array}$ & $\begin{array}{c}4,49 \\
\pm \\
0,27 \\
56\end{array}$ & $\begin{array}{c}85,55 \\
\pm \\
2,633 \\
1\end{array}$ & $\begin{array}{c}63,69 \\
\pm \\
7,837 \\
7\end{array}$ & $\begin{array}{c}48,03 \\
\pm \\
8,894 \\
7\end{array}$ & $\begin{array}{c}4,51 \\
\pm \\
0,27 \\
75\end{array}$ & $\begin{array}{c}4,51 \\
\pm \\
0,27 \\
77\end{array}$ & $\begin{array}{c}0,83 \\
\pm \\
0,07 \\
63\end{array}$ & $\begin{array}{c}66,63 \\
\pm \\
3,733 \\
9\end{array}$ \\
\hline
\end{tabular}

L: length, $\mathrm{W}$ : width, $\mathrm{T}$ : thickness, $\mathrm{D}_{\mathrm{a}}$ : arithmetic diameter, $\mathrm{D}_{\mathrm{g}}$ : geometric diameter, $\phi$ : sphericity, $\mathrm{S}$ : surface area, V: volume, $\mathrm{D}_{\text {sq }}$ : square mean diameter, $\mathrm{D}_{\mathrm{e}}$ : equivalent diameter, $\mathrm{R}_{\mathrm{as}}$ : seed aspect ratio, $\mathrm{m}_{1000}: 1000$ seed weight.

\section{Cluster analysis based on physical seed properties}

The dendrogram generated by physical seed properties is shown in figure 4. The dendrogram is composed of 2 major clusters at a distance of 189.16. Samples 23 and 34 being the first cluster. The second cluster is divided into two sub-clusters. IIa $(\mathrm{d}=34.72)$ is composed of accession 43 linked to accessions 55, 79, 41 and 6. IIb is further divided into 2 groups (IIb1, IIb2). IIb1 (d=27.49) is composed of samples 19, 91, 36, 65, 38, 35 and 15 one side clustered with samples $11,51,37,13,71,64,14,8,100,47$, $59,45,33,93,83,7$ and 5 another side. IIb2 (d=43.14) comprises sample 10 linked with two groups: the first one contains samples 90 and 74 . The second one is composed of two clades. The first calde $(\mathrm{d}=21.43)$ is composed of accessions 49, 72, 89, 42, 20, 29, 27, 46, 22, 102, 70, 56, 32, 77, 40, 26 and 18. The second clade $(\mathrm{d}=14.47)$ comprises samples $57,87,17,63,3,80$ and 
1. The higher distance of $\mathrm{d}=264$ is observed between samples 23 and 19 . High distances are also observed between sample 23 an all other accessions and between sample 34 and other accessions. In parallel, the lower distance $(\mathrm{d}=1)$ is observed between accessions 45 and 59. A distance of $\mathrm{d}=2$ is obtained for the following couples: 56-32, 26-40, 64-71.

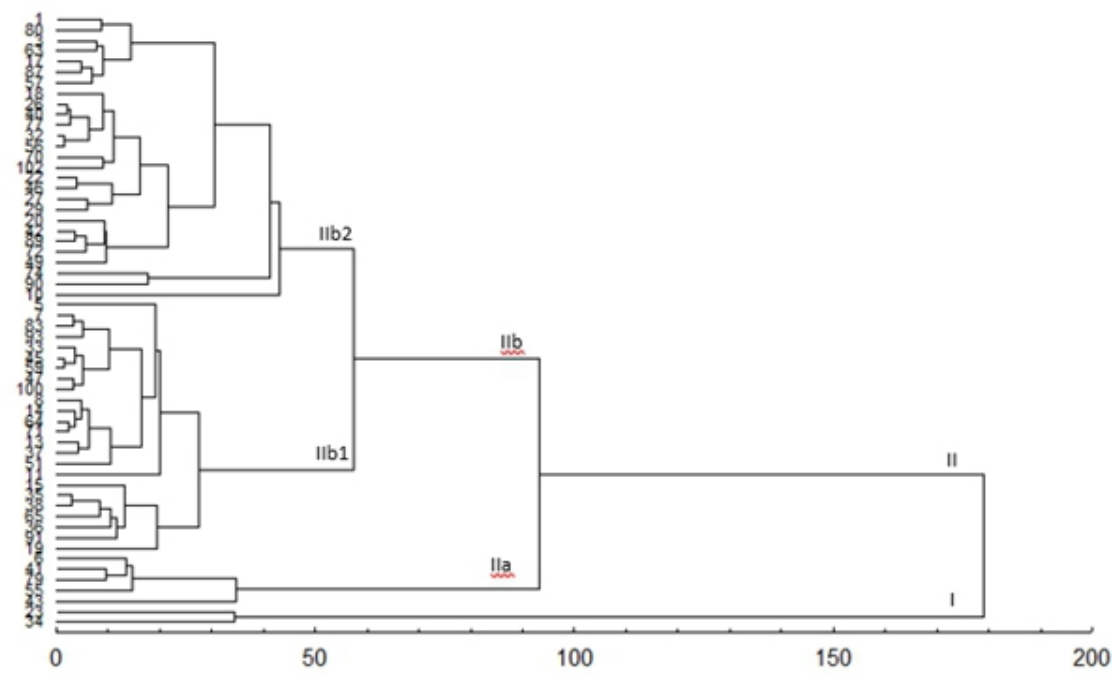

Figure 4. Dendrogram generated using UPGMA cluster analysis and Euclidean distances based on physical seed properties of accessions investigated

\section{Principal Component Analysis (PCA) of physical seed properties}

The results of PCA are shown in figure 5. The results revealed that the first three axes accounted for $94.95 \%$ of the total variation with $78.56 \%$ for PC1, $11.33 \%$ for PC2 and 5.05\% for PC3. Two-dimensional (2D) plot was obtained using the first two PCs. The seed properties which strongly contributed to the formation of PC1 are $\mathrm{D}_{\mathrm{e}}, \mathrm{D}_{\mathrm{a}}, \mathrm{D}_{\mathrm{g}}$ and $\mathrm{D}_{\mathrm{sq}}$ and those which most contribute to the formation of PC2 are $\mathrm{R}_{\text {as }}$ and $\phi$. In the other side, characters which have a low loading in accessions distinction are $\mathrm{R}_{\text {as }}$ and $\phi$ for $\mathrm{PC} 1$ and $\mathrm{V}$ and $\mathrm{T}$ for $\mathrm{PC} 2$. The strongest positive correlation was found between $D_{g}-D_{a}, D_{e}-D_{a}, D_{e}-D_{g}$ with $r=0.999$ followed by $D_{a}-D_{s q}$ and $D_{s q}-D_{e}$ with $r=0.993$. The strongest negative one was obtained between $L-\phi$ ( $r=-$ $0.532), \mathrm{D}_{\mathrm{sq}^{-}} \phi(\mathrm{r}=0.401)$ and $\mathrm{D}_{\mathrm{a}}-\phi(\mathrm{r}=0.400)$. 


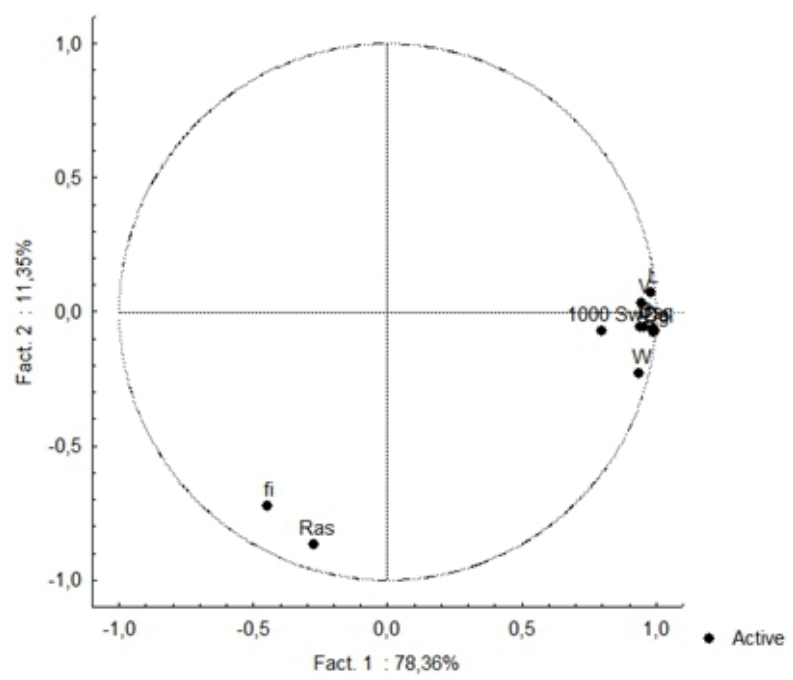

Figure 5. Principal Component Analysis based on 12 physical seed properties

\section{Correlation between enzymes patterns and physical seed properties}

Correlation between enzymatic systems data and physical seed properties was carried out with Mantel test based on Pearson's correlation. The p-value was calculated from the distribution of $\mathrm{r}(\mathrm{AB})$ using 10000 permutations. The test gives a value of $\mathrm{r}(\mathrm{AB} . \mathrm{C})=0.172$ showing a strong correlation between isozyms and seed properties since the p-value $(<0.0001)$ is below the significance level (5\%).

\section{Discussion}

\section{Enzymes polymorphism}

The electrophoretic mobility values in Table 2 may differ from those previously reported by other studies because of differences in the electrophoresis gel composition used, as reported by Jaaska (2008). The age of seedlings proved to be not important as it does not influence electrophoretic mobility. A strong correlation was obtained between data of the three studied systems one side and between the systems taken two by two one side which led utilization of one of them in taxa identification. Our data show that SOD is the most interspecific variable system, which could be related to its role in periferic metabolism. Accessions lacked the chloroplastic SOD-A even on the zymograms of leaflets, presumably because of the presence of an inhibitory substance as reported by Jaaska (2005). Our results concord with those reported by Gonzalez and ShiffinoWittman (1996) where eleven different SOD bands were found, ranged from 2 to 3 per sample, no one being common to all of them. Gels stained for Alcohol DeHydrogenase displayed three zones of activity. ADH is a dimeric protein encoded by two loci (ADH-A and ADH-B) according to the work of 
Li and Gao (1998). ADH isozymes should appear as dark bands on gels stained for ADH activity. SOD may appear as an artifact on gels stained with solutions containing MTT/PMS and it has been known to appear on gels stained for ADH after prolonged incubation. ADH and SOD data would be the same. Perhaps this contributed to the rather high correlation observed between the ADH and SOD data matrices. Aspartate Amino Transferase produced two monomorphic zones of activity with different intensities (Figure 2c). Each zone is specified by one gene: AAT-A and AAT-B that determined AAT isozymes. On the zymograms of green leaflets the AAT-A band, dominated in intensity, indicating possible plastidic nature. The following bands can be used as diagnostic characters for taxa discrimination by isozyme analyses: A38.2, AD33.5, S58, S41.6 for $V$. monantha subsp. cinerea, A27.6 for samples of sect. Cracca as in the cases of accessions 3, 26, 91 and 100, AD43, S49.2 for V. leucantha, AD31.6 and S39.5 for $V$. sativa subsp. obovata, S50.3 for V. narbonensis.

\section{Cluster analysis based on isozyms polymorphism}

There is a general agreement between the phenogram and taxonomic classification, with some exceptions. Cluster analysis of the three enzymatics systemes revealed two basic monophyletic groups: I) populations of $V$. sativa s.l (section Vicia) in one cluster II) all accessions of $V$. monantha (section Cracca) in one subcluster linked with samples of $V$. lutea (section Hypechusa) in a second subcluster which is connected to samples of $V$. narbonensis. Thus, species of sect. Narbonensis, (subgenus Vicia), sect. Hypechusa (subgenus Vicia) and sect. Cracca (subgenus Vicilla sensu Kupicha) clustered together.

\section{Cluster I}

Variability in all taxa of $V$. sativa is overlapping and many of the subdivisions are known to interbreed with each other (Bozkurt et al. 2013). In the present work, the all studied taxa of $V$. sativa (section Vicia) cluster together on the basis of isozyms polymorphism which indicates a close relationship between subspecies of $V$. sativa when it is difficult to determine distinct groups which could be individually identified as obovata, consobrina, cordata or angustifolia. According to Jaaska (2008), the subspecies of $V$. sativa have common orthozymes, differing mostly by the presence of additional allozymes of some heterozymes and in their relative occurrence. Our results are congruent with the AFLP data of Potokina et al. (2002) and Jaaska (2015) that showed all taxa of the V. sativa L. aggregate in a separate cluster. The same findings were reported in our prvious work on the same taxa using plant morphology (Bechkri and Khelifi 2016) and seed storage proteins polymorphism (not published). Concerning accession 17 
which was found to present lot of different morphological characters compared with the other accessions belonging to the same subspecies ( $V$. sativa subsp. obovata) wih the white standard (Bechkri and Khelifi 2016), it is connected to samples of the same taxa using isozyms data. Accessions of $V$. sativa which we attributed to subsp. cordata on the morphological ground could not be distinguished from other taxa of $V$. sativa by any specific morphs. The isozyms studied here can be used to discriminate taxa belonging to $V$. sativa at interpecific level but not at intraspecific one. Some accessions of $V$. sativa subsp. obovata were distinguished by having common SOD patern with morphs S37.5, S39.5 for samples 7 and 10 and morphs S34.5S39.5-S41 for samples 20, 22, 51, 57.

\section{Cluster II}

In the same group, samples of $V$. lutea (sect. Hypechusa) clustered all together as well as samples of $V$. narbonensis (sect. Narbonensis). The taxa of $V$. narbonensis showed the same three morphs (S37.5, S44.6, S50.3). Our results concord with those of Jaaska and Leht (2007) which showed the species of sections Hypechusa as sister to the clade of section Narbonensis. A closer relationship between the NSC and section Hypechusa has been deduced based on isozyme studies by Jaaska (1997) where the Narbonensis section appears as a clade on both cladistic and phenetic trees which is linked to species of the section Hypechusa. Jaaska (2005) reported that species of the section Cracca form a subgroup of closely related species that revealed extensive homologous polymorphism with shared allozymes without any differentiation by species-specific orthozymes. Vicia monantha appears basally sister to the remaining species of the section on the phylogenetic tree. Also, in our rsults, the cluster II contains samples of $V$. monantha subsp. calcarata (sect. Cracca) all together joined with the two samples of $V$. tenuifolia and the unique sample of $V$. leucantha. In spite of this, our analysis of enzyms data supports Kupicha's placement of $V$. leucantha in section Cracca as was also done by Davis and Plitmann (1970). The same findings were reported in our previous work using plant morphology (not published). The species $V$. monantha, $V$. tenuifolia and $V$. leucantha, classified by Kupicha (1976) in section Cracca appear in a well-supported monophyletic group in the present work. V. tenuifolia and V. leucantha, form a subgroup of closely related species that revealed extensive homologous polymorphism with shared characters. The sample 46 belonging to $V$. monantha subsp. cinerea forms a separate subcluster as it has a unique pattern for each of the three studied systems. It is not linked to accession 91 of the same taxa which is clustered with accessions of $V$. lutea. This rare variante may be ignored in aphylogenetic analysis as proposed by Stevens (1991). The unexpected linkage of the sample 91 with samples of $V$. lutea in the cladistic isozyme 
tree may be caused by electrophoretic homoplasy of some shared orthozymes as reported by Jaaska (2015). The three systems studied seem to be more suitable for studies of interspecific genetic diversity than of intraspecific affinities.

\section{Physical seed properties}

The variability of physical seeds properties of natural populations of Vicia in Algeria has not previously been characterized. In many cases, morphological characteristics such as seed dimensions can be used to distinguish species and subspecies (Bewley and Black, 1994; Hammet et al., 1996). The relationship between seed size and seed number may be an important mechanism underlying the abundance and dynamics of plant species (Jakobsson and Eriksson, 2000). Our UPGMA cluster method gave no clear grouping. The general picture showed that accessions of the same species preferred to cluster with themselves rather than have a wide distribution in different groups. The accessions of species traditionally considered to have a high degree of variation (Ball 1968), for example $V$. sativa, were present in more than one cluster group. The same observations were done by Perrino et al. (1984). Physical seed properties which separate the most the samples are equivalent diameter, arithmetic diameter, geometric diameter and square diameter. At the same time, strong correlation was found between these four properties showing the potential use of one of them to distinguish taxa. Our results show that both the arithmetic mean and the geometric mean method can be used to determine the average diameter of vetch seeds. A similar result was found by Kaleemullah and Gunasekar (2002) for arecanut kernels. Results show that the longer, wider and thicker seed can be the less spherical as in the case of sample 6 ( $V$. sativa subsp. obovata). Values of seeds surface area and volume follow those of size dimensions. Samples 19 and 23 are a good example in this case. Concerning variability between samples belonging to the same taxa, the explanation for this phenomenon lies in the genetic and environmental characteristics of seed polymorphism (Bewley and Black, 1994). In species producing two or more seed types, there is a tendency for seed functions to diverge, each type being adapted to an aspect of the environment to which it is predisposed, whilst being buffered by the other seed type (Mandak, 1997). Moreover, it has been proven that seeds which vary in appearance also vary in chemical composition (Aniszewski et al. 2001). This suggests that if we are to manipulate the chemical constituents of seeds in general, it is very important to classify seeds according to types and physical properties. According to Westoby et al. (1992), selection pressures acting on the seeds during the course of an experiment can influence their size, number and weight. Several studies on seed characterestics (Getinet and Rakow 1997; Takashi 1997; 
Kehinde et al. 1997; Asiedu and Powell, 1998) support the fact that seed polymorphism has a genetic basis. suggested that morphological characteristics of seeds such as seed shape can be used to distinguish taxa. In the case of Vicia sativa, the average seed dimensions decreased from $V$. sativa subsp. obovata to $V$. sativa subsp. angustifolia passing by $V$. sativa subsp. cordata and $V$. sativa subsp. consobrina. These results are more detailed than those given in the flora of Algeria (Quézel and Santa 1962) in which, the authors used seed dimension to discriminate subspecies of $V$. sativa. Indeed, they give a range of 3-5 mm for subsp. obovata and 2-3 mm for subsp. cordata, consobrina and angustifolia with any distinction between these three subspecies by seed dimensions. Species which produce two or more seed dimensions represent groups in which divergent strategies, usually present in different taxa, are combined in one individual (Mandak, 1997). Our results confirm the fact that $V$. sativa contains different sub-species often mentioned by taxonomists. For $V$. monantha, our results show that the two subspecies (calcarata and cinerea) have close values regarding physical properties studied and join Quézel and Santa (1962) who did not use seed dimensions to discriminate between the tow subspecies. The same conclusion can be given for $V$. lutea subsp. eu-lutea and V. lutea subsp. vestita which are not distinguish by seed dimensions but by the flower colour. Seed dimensions were not considered in the flora to distinguish $V$. tenuifolia and V. leucantha.

\section{Conclusion}

The species groupings using AAT, SOD and ADH patterns are consistent with traditional taxonomic species delimitation. Therefore, isozyme patterns are useful and reliable biochemical markers for the taxonomic delimitation and characterization of Vicia germplasm. Evidently, additional data as evidence from the DNA characters are needed for more sound phylogenetic and taxonomic conclusions. In another side, due to the fact that classification of seeds is of major technical and economical importance in the agricultural industry, discriminant analysis was used to classify the seeds but shows no correspondence with systematics. In order to design equipment and facilities for the handling, conveying, separation, drying, aeration, storing and processing of vetch seed, future studies should be conducted to determine the 12 physical properties studied as a function of moisture content.

Financial disclosure: This work is not supported by any financial assistance. Conflict of interest: The authors declare that they have no conflicts of interest. 


\section{References:}

1. Aniszewski T, Kuparihm, Leinonen AJ. 2001. Seed Number, Seed Size and Seed Diversity in Washington Lupin (Lupinus polyphyllus Lindl.). Annals of Botany. 87: 77-82

2. Asiedu EA, Powell AA. 1998. Comparisons of the storage potential of cultivars on cowpea (Vigna unguiculata) differing in seed coat pigmentation. Seed Science and Technology. 26: 211-221.

3. Asoegwu SN, Ohanyere SO, Kanu OP, Iwueke CN. 2006. Physical properties of African Oil Bean Seed (Pentaclethra macrophylla). Agricultural Engineering International: the CIGR Ejournal. Vol. VII.

4. Ball PW. 1968. Vicia L. In: Tutin TG, Heywood VH, Burges NA, Moore DM, Valentine DH, Walters SM, Webb DA. (eds.) Flora Europaea, vol.2, Rosaceae to Umbelliferae. Cambridge University Press, Cambridge, pp. 129-136

5. Bechkri S, Khelifi D. 2016. Variation in Vicia sativa s.1. from Algeria based on morphological characters and ecogeographic parameters. Genetic Resources and Crop Evolution DOI 10.1007/s10722-0160404-1

6. Bewley JD, Black M. 1994. Seeds. Physiology of development and germination. New York, London: Plenum Press.

7. Bozkurt M, Ertugrul K, Uysal T. 2013. The determination of genetic relationships among some Vicia L. (Vetch) taxa by using ISSR markers. Biological Diversity and Conservation. 6/3: 135-139

8. Davis PH, Plitmann U. 1970. Vicia L. In: Davis PH. (ed.) Flora of Turkey and the East Aegean Islands. University Press, Edinburgh, pp. 274-321

9. Gates P, Boulter D. 1980. The use of pollen isoenzymes as an aid to the breeding of field beans (Vicia faba L.). New phytologist. 84(3): 501-504

10. Getinet A, Rakow G. 1997. Repression of seed coat pigmentation in Ethiopian mustard. Canadian Journal of Plant Science. 77: 501-505.

11. Gonzalez AC, Shiffino-Wittman MT. 1996. Isoenzymatic characterization of some Lathyrus L. and Vicia L. speices (Leguminosae) occurring in Rio Grande do Sul (Southern Brazil). Brazilian Journal of Genetics. 19(2): 283-288

12. Hammett KRW, Murray BG, Markham KR, Hallett IC, Osterloh I. 1996. New interspecific hybrids in Lathyrus (Leguminosae): Lathyrus annuus $x$ L. hierosolymitanus. Botanical Journal of the Linnean Society. 122: 89-101.

13. Hanelt P, Mettin D. 1989. Biosystematics of the genus Vicia L. (Leguminosae) Annu. Rev. Ecol. Syst. 20: 199-223 
14. Hauhouot-O’Hara M, Criner BR, Brusewitz GH, Solie JB. 2000. "Selected Physical Characteristics and Aerodynamic Properties of Cheat Seed for the Separation from Wheat". Agricultural Engineering International: the CIGR Journal of Scientific Research and Development. Vol. II.

15. Iannelli P. 1964. Variety testing of vetches. -Proc. Internatl. Seed Test. Assoc. 29: 887-907

16. Jaaska V. 2008. Isozyme evidence on the specific distinctness and phylogenetic position of Vicia incisa (Fabaceae). Centr. Eur. J. Biol. 3: 169-176.

17. Jaaska V. 2015. Phylogenetic relationships among sections Vicia, Sepium and Lathyroides of Vicia subgenus Vicia: isozyme evidence. Biochemical Systematics and Ecology. 62: 186-193

18. Jaaska V. 1997. Isoenzyme diversity and phylogenetic affinities in Vicia subgenus Vicia (Fabaceae). Genet. Res. Crop Evol. 44: 557574

19. Jaaska V, Leht M. 2007. Phylogenetic relationships between and within sections Hypechusa, Narbonensis and Peregrinae of genus Vicia (Fabaceae) based on evidence from isozymes and morphology. CEJB 2(1): 137-155

20. Jaaska V. 2001. Isoenzyme diversity and phylogenetic relationships among the American beans of the genus Vigna Savi (Fabaceae). Biochemical Systematics and Ecology. 29: 545-554.

21. Jaaska V. 2005. Isozyme Variation and Phylogenetic Relationships in Vicia subgenus Cracca (Fabaceae). Annals of Botany. 96: 1085-1096

22. Jakobsson A, Eriksson O. 2000. A comparative study of seed number, seed size, seedling size and recruitment in grassland plants. Oikos. 88: 494-502.

23. Kaleemullah S, Gunasekar JJ. 2002. Moisture-dependent physical properties of arecanut trues. Biosys. Eng. 82: 331-338

24. Kehinde OB, Myers GO, Fawole I. 1997. Analysis of genetic linkage in the cowpea Vigna unguiculata. Pertanika Journal of Tropical Agricultural Science. 20: 75-82.

25. Kupicha FK. 1976. The infrageneric structure of Vicia. Notes Roy. Bot. Gard. Edinburgh. 34: 287-326

26. Leht M, Jaaska V. 2002. Cladistic and phenetic analysis of relationships in Vicia subgenus Vicia (Fabaceae) by morphology and isozymes. Plant Systematics and Evolution. 232: 237-260.

27. Li R, Gao Y. 1998. Biochemical Genetic Studies on Wild Populations of the Vicia amoena Complex (Tribe Vicieae: Fabaceae). Biochemical Genetics. 36 (5/6): 213-217 
28. Mandak B. 1997. Seed heteromorphism and the life cycle of plants: a literature review. Preslia-Prague. 69: 129-159.

29. Mantel N. 1967. Adaptation of Karber's method for estimating the exponential parameter from quantal data, and its relationship to birth, death, and branching processes. Biometrics. 23: 739-746

30. Maxted N. 1993a. A phenetic investigation of Vicia L. subgenus Vicia (Leguminosae, Vicieae). Bot. J. Linn. Soc. 111: 155-182.

31. Mohsenin NN. 1970. Physical properties of plant and animal materials. Gordon and Breach Sci. Publishers, New York

32. Olajide JD, Ade-Omowaye BIO. 1999. Some physical properties of locust bean seed. J. Agric. Eng. Res. 74: 213-215

33. Perrino P, Yarwood M, Hanelt P, Polignano GB. 1984. Variation of seed characters in selected Vicia species. Kulturpflanze. 32: 103-122.

34. Potokina E, Tomooka N, Vaughan DA, Alexandrova T, Xu RQ. 1999. Phylogeny of Vicia subgenus Vicia (Fabaceae) based on analysis of RAPDs and RFLP of PCR-amplified chloroplast genes. Genet Res. Crop Evol. 46: 149-161

35. Potokina E, Blattner R.F, Alexandrova T, Bachmann K. 2002. AFLP diversity in the common vetch (Vicia sativa L.) on the world scale. Theor. Appl. Genet. 105 : 58-67

36. Quézel P, Santa S. 1962. Nouvelle flore de 1'Algérie et des régions méridionales. Tome 1. Editions du centre national de la recherche scientifique. Paris, pp. 524- 531

37. Scherer R, Kutzbach HD. 1978. Mechanische Eigenschaften von Koerner-fruechten [Mechanical properties of grains]. Grundlage der Landtechnik. 28(1): 6-12.

38. Seifi MR, Alimardani R. 2010. The moisture content effect of some physical and mechanical properties of corn. Journal of Agric. Science, Canadian Centre of Science and Education. 2(4):125-134

39. Stevens PF. 1991. Character states, morphological variation and phylogenetic analysis: a review. Syst. Bot. 16: 553-583

40. Subukola OP, Onwuka VI. 2011. Effects of moisture content on some physical properties of locust bean seed (Parkiafillicoidea). Journal of Food Process Engineering. 34: 1947-1961.

41. Suso MJ, Moreno MT. 1986. Isoenzymatic polymorphism of superoxide dismutase (SOD) in Vicia faba and its systematic implication. FABIS Newslett. 16: 3-11

42. Takashi R. 1997. Association of soybean genes I and T with lowtemperature induced seed coat deterioration. CropScience. 37:17551759.

43. Taser OF, Altuntas E, Ozgog. 2005. Physical properties of Hungarian and common vetch seeds. J. Applied Sci. 5: 323-326 
44. Wendel JF, Weeden NF. 1989. Visualization and interpretation of plant isozymes. I $n \mathrm{~S}$ o $1 \mathrm{t}$ i s D E. Soltis PS. Isozymes in plant biology, p. 5-44. Portland, Oregon: Dioscorides Press.

45. Westoby M, Jurado E, Leishman M. 1992. Comparative evolutionary ecology of seed size. Trends in Ecology and Evolution. 7: 368-372.

46. Wolff G. 1980. Investigations on the relations within the family Papilionaceae on the basis of electrophoretic banding patterns. Theor. Appl. Genet. 57: 225-232

47. Wu S, Sokhansanj S, Fang R, Opoku A. 1999. Influence of physical properties and operating conditions on particle segregation on gravity table. Appl. Eng. Agric. 15(5): 495-499.

48. Yamamoto K. 1979. Estimation of genetic homogeneity by isoenzymes from interspecific hybrids progeniesbetween Vicia amphicarpa, true and V. macrocarpa. Japan. J. Breed. 29: 59-65

49. Yamamoto K. 1986. Interspecific hybridization among Vicia narbonensis and its rlated species. Biol? Zentralbl. Leipziig.

50. Yamamoto K, Plitmann U. 1980. Isozyme polymorphism in species of the genus Vicia (Leguminosae) Japanese Journal of Genetics. 55: $151-164$

51. Zewdu AD. 2004. Untersuchungen zur Leistungssteigerung der Reinigungsanlage eines Maehdreschers durch zusaetzlichen Querschwingungen (Investigations into performance improvement of combine cleaning shoe through additional lateral oscillations). [PhD Thesis]. Dresden, Germany: Technical University of Dresden. 125 p. 